TRANSACTIONS OF THE

AMERICAN MATHEMATICAL SOCIETY

Volume 363, Number 6, June 2011, Pages 3133-3165

S 0002-9947(2011)05262-1

Article electronically published on January 27, 2011

\title{
SOLUTION OF THE TRUNCATED MOMENT PROBLEM WITH VARIETY $y=x^{3}$
}

\author{
LAWRENCE A. FIALKOW
}

\begin{abstract}
We show that positivity, consistency, and the variety condition, which are sufficient to solve the truncated moment problem on planar curves of degree 2, are not sufficient for curves of higher degree. Using new, partly algorithmic, conditions based on positive moment matrix extensions, we present a concrete solution to the truncated moment problem on the curve $y=x^{3}$. We also use moment matrix extensions to solve (in a less concrete sense) truncated moment problems on curves of the form $y=g(x)$ and $y g(x)=1(g \in \mathbb{R}[x])$, leading to degree-bounded weighted sum-of-squares representations for polynomials which are strictly positive on such curves.
\end{abstract}

\section{INTRODUCTION}

Let $\beta \equiv \beta^{(2 n)}=\left\{\beta_{i}\right\}_{i \in \mathbb{Z}_{+}^{d},|i| \leq 2 n}$ denote a real $d$-dimensional multisequence of degree $2 n$, and let $K$ denote a closed subset of $\mathbb{R}^{d}$. The truncated $K$-moment problem for $\beta$ concerns the existence of a positive Borel measure $\mu$, supported in $K$, such that

$$
\beta_{i}=\int_{K} x^{i} d \mu\left(i \in \mathbb{Z}_{+}^{d},|i| \leq 2 n\right) .
$$

(Here, for $x \equiv\left(x_{1}, \ldots, x_{d}\right) \in \mathbb{R}^{d}$ and $i \equiv\left(i_{1}, \ldots, i_{d}\right) \in \mathbb{Z}_{+}^{d}$, we set $|i|=i_{1}+\cdots+i_{d}$ and $x^{i}=x_{1}^{i_{1}} \cdots x_{d}^{i_{d}}$.) A measure as in (1.1) is a $K$-representing measure for $\beta$; for $K=\mathbb{R}^{d}$, we refer to $\mu$ simply as a representing measure. Two general, but abstract, solutions to the truncated $K$-moment problem are known; one involves flat extensions of positive moment matrices [CF6 (cf. Theorem 1.8 below for $K=\mathbb{R}^{d}$ ) and the other entails extensions of $K$-positive linear functionals [CF8] (see Section $6)$.

By a concrete solution to the truncated $K$-moment problem we mean a set of conditions for $K$-representing measures that can be effectively tested in numerical examples. Concrete solutions to the truncated $K$-moment problem are known in only a few cases: for $d=1$, with $K=\mathbb{R},\left[0,+\infty\right.$ ), or $\bigcup_{i=1}^{m}\left[a_{i}, b_{i}\right]$ (cf. $[\mathrm{AK}]$, $[\mathrm{KN}$, CF1), and for $d=2$, when $K$ is an algebraic curve $p(x, y)=0$ with $\operatorname{deg} p \leq 2$ (cf. [CF7, Theorem 1.2 (below)). In the sequel we provide a concrete solution in

Received by the editors April 19, 2008 and, in revised form, July 10, 2009.

2000 Mathematics Subject Classification. Primary 47A57, 47A20, 44A60; Secondary 15A57, $15-04,47 \mathrm{~N} 40$.

Key words and phrases. Truncated moment problems, moment matrix extensions, representing measures, positive polynomials.

This research was partially supported by NSF Research Grants DMS-0457138 and DMS0758378 . 
the case when $K$ is the planar curve $y=x^{3}$ (cf. Theorem 1.4). Our results show for the first time that the positivity and variety conditions of $[\mathrm{AK},[\mathrm{KN}$, [CF1, [CF7, CFM] are inadequate to resolve truncated $K$-moment problems on curves of higher degree (cf. Theorem 1.3). Instead, motivated by a recent generalization of Tchakaloff's Theorem due to Bayer and Teichmann [BT], we develop new conditions for representing measures based on positive moment matrix extensions. In some cases, these extensions can be detected analytically (cf. Theorem 1.1-iv)), while in other cases they must be determined algorithmically (cf. Theorem 1.4-iii)).

For the classical full moment problem concerning $\beta^{(\infty)} \equiv\left\{\beta_{i}\right\}_{i \in \mathbb{Z}_{+}^{d}}$ (cf. [Akh], $[\mathrm{ST}]$ ), a theorem of Stochel [S2] shows that $\beta^{(\infty)}$ has a $K$-representing measure if and only if for each $n, \beta^{(2 n)}$ has a $K$-representing measure. In this sense, solving the truncated $K$-moment problem also solves the full $K$-moment problem. For a semialgebraic closed set $K$, [CF6, Cor. 1.4] solves the truncated $K$-moment problem in terms of flat, i.e., rank-preserving, extensions of positive moment matrices and localizing matrices. Flat extensions are, however, problematic. For example, it is still unknown whether, for $d=2$, a positive definite $M(2)$ admits a flat extension $M(3)$. For this reason, it is desirable to identify cases where the requirement for flat extensions can be relaxed. In Section 6 , we solve the truncated $K$-moment problem in terms of positive moment matrix extensions, but without the requirement for flat extensions, for planar curves of the form $y=g(x)$ and $y g(x)=1(g \in \mathbb{R}[x])$. This solution is not really "concrete" (because we do not have precise conditions for the required extensions), but it is strong enough to recover Stochel's solution in [S1] to the full $K$-moment problem for these curves, and it also yields "degree-bounded" weighted sum-of-squares decompositions for polynomials that are strictly positive on these curves (cf. Proposition 6.1 and Remark 6.5).

Let $M(n) \equiv M(n)(\beta)$ denote the moment matrix associated with $\beta$ (see below for terminology and notation). The rows and columns of $M(n)$ are denoted by $X^{i}$ and are indexed (in degree-lexicographic order) by the monomials $x^{i}$ in $\mathcal{P}_{n} \equiv\{p \in$ $\left.\mathbb{R}\left[x_{1}, \ldots, x_{d}\right]: \operatorname{deg} p \leq n\right\}$. Corresponding to $p \equiv \sum_{i \in \mathbb{Z}_{+}^{d},|i| \leq n} a_{i} x^{i} \in \mathcal{P}_{n}$ is the element $p(X) \equiv \sum a_{i} X^{i}$ of $\mathcal{C}_{M(n)}$, the column space of $M(n) ; M(n)$ is recursively generated if whenever $p, q, p q \in \mathcal{P}_{n}$ and $p(X)=0$, then $(p q)(X)=0$. Positivity and recursiveness of $M(n)$ are necessary conditions for representing measures [CF1], CF3. For $p \in \mathcal{P}_{n}, M(n)$ is $p$-pure if the only dependence relations in $\mathcal{C}_{M(n)}$ are those of the form $(p q)(X)=0\left(q \in \mathcal{P}_{n-\operatorname{deg} p}\right)$. Let $p(x, y)=y-x^{3}$ and let $\Gamma$ denote the curve $y=x^{3}$. Our main result, which follows, characterizes the existence of $\Gamma$-representing measures in the $p$-pure case.

Theorem 1.1. Suppose $d=2, n \geq 3, p(x, y)=y-x^{3}$, and $M(n)$ is p-pure. The following are equivalent for $\beta \equiv \beta^{(2 n)}$ :

i) $\beta$ has a representing measure (necessarily supported in $y=x^{3}$ );

ii) $\beta$ has a rank $M(n)$-atomic $\Gamma$-representing measure;

iii) $M(n)$ admits a positive, recursively generated moment matrix extension $M(n+1)$

iv) (concrete condition) $M(n) \succeq 0$ and $\beta_{1,2 n-1}>\psi(\beta)$ (where $\psi(\beta)$ is the rational expression in the moment data defined by (2.21) below).

Theorem 1.1 is proved in Section 2. The numerical condition $\beta_{1,2 n-1}>\psi(\beta)$ in (iv) is a new type of condition in truncated moment theory, one that is intimately related to the curve $y=x^{3}$. No such condition is required for planar curves of 
degree 1 or 2 (cf. Theorem 1.2 below). For curves of degree 3 or higher, conditions (or sets of conditions) similar to (iv) will be required, but the specific conditions will depend on the curves themselves. More generally, the moment matrix techniques that we employ can be adapted to general curves, but certain details of our proofs seem to apply only to $y=x^{3}$. For this reason, our results suggest that a solution to the truncated moment problem for planar curves cannot be both general and concrete. We discuss this issue in Question 1.5 and in Remark 2.5.

Returning to the general case $(d \geq 1)$, let $\mathcal{V} \equiv \mathcal{V}(M(n))$ denote the algebraic variety corresponding to $M(n)$, i.e., $\mathcal{V}=\bigcap_{p \in \mathcal{P}_{n}, p(X)=0} \mathcal{Z}_{p}$ (where $\mathcal{Z}_{p}=\{x \in$ $\left.\left.\mathbb{R}^{d}: p(x)=0\right\}\right)$. A necessary condition for representing measures is the "variety condition", $r \equiv \operatorname{rank} M(n) \leq v \equiv \operatorname{card} \mathcal{V}(M(n))$ (cf. [CF3, pg. 182]). The following result of [CF7] provides a concrete solution to the truncated $K$-moment problem for the case when $K$ is a planar curve of degree 1 or 2 .

Theorem 1.2 ([CF7, Theorem 1.2]; cf. [F3]). Suppose $d=2$ and $1 \leq \operatorname{deg} p(x, y) \leq$ 2. For $n \geq \operatorname{deg} p$, the following are equivalent for $\beta \equiv \beta^{(2 n)}$ :

i) $\beta$ has a representing measure supported in $\mathcal{Z}_{p}$;

ii) $M(n)$ has a column dependence relation $p(X, Y)=0$, and $M(n)$ admits a positive, recursively generated extension $M(n+1)$;

iii) (concrete condition) $M(n)$ is positive and recursively generated, $r \leq v$, and $p(X, Y)=0$ in $\mathcal{C}_{M(n)}$.

In [CFM, Theorem 5.2], Curto, Möller, and the author proved that Theorem 1.2iii) does not extend to polynomials of degree 3 , e.g., it does not extend to $p(x, y)=$ $y-x^{3}$ : there exists $M(3)$ that is positive and recursively generated, with $Y=X^{3}$ in $\mathcal{C}_{M(3)}$ and $r=v=8$, such that $\beta^{(6)}$ has no representing measure. Consider the Riesz functional $\Lambda_{\beta}: \mathcal{P}_{2 n} \longrightarrow \mathbb{R}$ defined by $\Lambda_{\beta}\left(\sum_{i \in \mathbb{Z}_{+}^{d},|i| \leq 2 n} a_{i} x^{i}\right)=\sum a_{i} \beta_{i}$. Following CFM], we say that $\beta$ is consistent if $p \in \mathcal{P}_{2 n}, p \mid \mathcal{V} \equiv 0 \Longrightarrow \Lambda_{\beta}(p)=0$. Consistency is a necessary condition for representing measures that is strictly stronger than recursiveness (cf. [CFM, Theorem 5.2]). In [CFM] we proved that for $d \geq 1$, if $M(n)$ is extremal, i.e., $r=v$, then $\beta$ has a representing measure if and only if $M(n) \succeq 0$ and $\beta$ is consistent. In CFM, Question 1.1] we asked whether this result can be extended to the setting where $r \leq v$. The following result uses Theorem 1.1 to resolve this question.

Theorem 1.3. Let $d=2$. There exists $M(3)$ that is positive and consistent, with column relation $Y=X^{3}$ and $r<v$, such that $\beta^{(6)}$ admits no representing measure.

The proof of Theorem 1.3 is established by Example 3.2, which shows that besides positivity, consistency, and the variety condition, representing measures sometimes entail an auxiliary condition such as that in Theorem 1.1-iv).

The analysis of the truncated $\Gamma$-moment problem in Theorem 1.4 (below) depends in part on the notion of recursively determinate moment matrices. For this class, the existence of representing measures can always be determined algorithmically. Recall from [F4] that $M(n)$ is recursively determinate if the column dependence relations in $M(n)$ completely determine the existence or nonexistence of (necessarily unique) successive positive, recursively generated extensions $M(n+1)$, $M(n+2), \ldots$ (as described by Theorem 1.6 (below) and [F4, Algorithm 4.10]). These extensions in turn determine the existence of representing measures for $\beta^{(2 n)}$. We refer to such extensions as recursively determined extensions. The basic example of recursive determinancy is the case of flat data, where $M(n) \succeq 0$ and 
rank $M(n)=\operatorname{rank} M(n-1)$. In this case, for each $i \in \mathbb{Z}_{+}^{d}$ with $|i|=n$, there exists $p_{i} \in \mathcal{P}_{n-1}$ such that $X^{i}=p_{i}(X)$ in $\mathcal{C}_{M(n)}$. CF2 implies that for such $M(n)$, there exists a unique rank-preserving, recursively generated moment matrix extension $M(n+1)$ (and a corresponding representing measure), determined by $X^{i+j}=\left(x^{j} p_{i}\right)(X)\left(i, j \in \mathbb{Z}_{+}^{d},|i|=n,|j|=1\right)$ (cf. Theorem 1.6 below). It follows from [F4, Proposition 4.2] that if $d=2$ and $M(n)$ is recursively generated, with $Y=X^{3}$ in $\mathcal{C}_{M(n)}$, then $M(n)$ is recursively determinate if and only if there is a column relation of the form $Y^{i+1}=\alpha X^{2} Y^{i-1}+\gamma X Y^{i}+q(X, Y)$ for some $i$, $2 \leq i<n$, and some $q \in \mathcal{P}_{i}$.

Our solution to the truncated $\Gamma$-moment problem, which follows in Theorem 1.4 (below), is based on a classification of the dependence relations in the columns of $M(n)$. Suppose $M(n)$ is positive and recursively generated, with the column relation $Y=X^{3}$. Part i) of Theorem 1.4 concerns the case when the first 6 columns of $M(n)$, corresponding to the monomials $1, x, y, x^{2}, x y, y^{2}$, are linearly dependent. This is the case when a $\Gamma$-representing measure, if one exists, is necessarily contained in the intersection of $y=x^{3}$ with some curve of degree 1 or 2 . This case follows directly from Theorem 1.2. Suppose now that the first 6 columns of $M(n)$ are independent; equivalently, $M(2) \succ 0$. Part ii) of Theorem 1.4 concerns the case when $M(n)$ is $\left(y-x^{3}\right)$-pure, i.e., the column dependence relations in $M(n)$ are precisely those of the form $q(X, Y)\left(Y-X^{3}\right)=0$ for $q \in \mathcal{P}_{n-3}$. This case is treated by Theorem 1.1 (cf. Section 2). Part iii) of Theorem 1.4 concerns the remaining case, when $M(2) \succ 0$ and $Y=X^{3}$, but there is some column dependence relation that cannot be obtained from $Y=X^{3}$ via recursiveness. In this case, there is a minimal $i, 2 \leq i<n$, such that exactly one of the following three conditions holds:

$$
\begin{gathered}
X^{2} Y^{i-1}=q(X, Y) \text { for some } q \in \mathcal{P}_{i} ; \\
(1.2) \text { does not hold, but } \\
X Y^{i}=\alpha X^{2} Y^{i-1}+q(X, Y) \text { for some } \alpha \in \mathbb{R}, q \in \mathcal{P}_{i} ; \\
(1.2) \text { and }(1.3) \text { do not hold, but } \\
Y^{i+1}=\alpha X^{2} Y^{i-1}+\gamma X Y^{i}+q(X, Y) \text { for some } \alpha, \gamma \in \mathbb{R}, q \in \mathcal{P}_{i} .
\end{gathered}
$$

If (1.2) holds, the existence of a representing measure will be derived from the Flat Extension Theorem of [CF6] (cf. Theorem 1.6 below). If (1.3) holds, the existence of a representing measure will be derived in Section 4 (Theorem 4.1). Finally, if (1.4) holds, we will show that $M(n)$ is recursively determinate, so the existence or nonexistence of a representing measure can be determined by the algorithm of [F4] (cf. Theorem 1.7 below). As described in Theorem 1.4, at most 2 iterations of the algorithm are required. Our solution to the truncated $\Gamma$-moment problem now assumes the following form.

Theorem 1.4. Let $n \geq 3$ and let $p(x, y)=y-x^{3}$. If $\beta \equiv \beta^{(2 n)}$ has a $\Gamma$-representing measure, then $M(n)$ is positive and recursively generated, satisfies the variety condition, and has the column relation $Y=X^{3}$. Conversely, suppose $M(n)$ satisfies the preceding conditions, so that any representing measure is necessarily supported in $\Gamma$.

i) If $M(2)$ is singular, then there is a representing measure (cf. Theorem 1.3).

ii) If $M(n)$ is $p$-pure, then there is a representing measure if and only $\beta_{1,2 n-1}>$ $\psi(\beta)$ (cf. Theorem 1.1). 
iii) If $M(n)$ satisfies neither the hypothesis of i) nor of ii), then exactly one of (1.2)-(1.4) holds. If (1.2) or (1.3) holds, there is a representing measure. If (1.4) holds, then $M(n)$ is recursively determinate. If (1.4) holds with $i<n-2$, there is a representing measure; if $i=n-2$, there is a measure if and only if rank $M(n)=\operatorname{rank} M(n-1)$, or the recursively determined extension $M(n+1)$ satisfies rank $M(n+1)=\operatorname{rank} M(n)$; if $i=n-1$, there is a measure if and only if there are positive, recursively determined extensions $M(n+1)$ and $M(n+2)$, with $\operatorname{rank} M(n+1)=\operatorname{rank} M(n)$ or $\operatorname{rank} M(n+2)=\operatorname{rank} M(n+1)$.

We summarize the organization of the paper as follows. Section 1 contains statements of the main results and also contains some background material on the truncated $K$-moment problem. Section 2 contains the proof of the main result, Theorem 1.1, which is used in Section 4 to prove Theorem 1.4-ii). The results of Section 2 are also used in Section 3 to prove Theorem 1.3. Section 4 contains the proof of the case of Theorem 1.4 described by (1.3). Section 5 contains the complete proof of Theorem 1.4, using results from Sections 2 and 4; this solves the $\Gamma$-moment problem for $n \geq 3$. The second part of Section 5 treats the $\Gamma$ moment problem for $n=1$ and $n=2$. Section 6 is largely independent of the rest of the paper and describes new cases where the truncated moment problem can be solved entirely in terms of positive moment matrix extensions (and without the need for a "flat" extension as in Theorem 1.8). The results of Section 6 are of "intermediate" concreteness, more concrete than the general, but abstract, solutions to the truncated moment problem in Theorem 1.8 or CF8, but less concrete than Theorem 1.4.

The conditions of Theorem 1.4 are "concrete" in the following sense. Positivity and recursiveness can be readily checked using elementary linear algebra. The variety condition may require the solution of polynomial equations. For moderate values of $n$, this can be carried out with software such as Mathematica, MatLab, etc. Finally, it is a simple matter to determine whether $M(2)$ is singular, whether $M(n)$ is $p$-pure, or whether one of (1.2)-(1.4) holds. If (1.4) holds, then $M(n)$ is recursively determinate, and the existence or nonexistence of a representing measure can be resolved by Theorem 1.7 with $0 \leq v-r \leq 1$. Thus, in this case, it is only necessary to use [F3, Algorithm 4.10] to construct, at most, the recursively determined extensions $M(n+1)$ and $M(n+2)$. Example 5.2 illustrates the existence of a representing measure in this case, and [CFM], Theorem 5.2] and [F4, Example 4.18] illustrate the nonexistence of representing measures in this case. The cases of the truncated $\Gamma$-moment problem for $\beta^{(2 n)}$ with $n=1$ or $n=2$ require special arguments given at the end of Section 5 .

We note a distinction between Theorem 1.4 and many of the results in the moment literature. The full $K$-moment problem for $\beta^{(\infty)}$ has been solved concretely

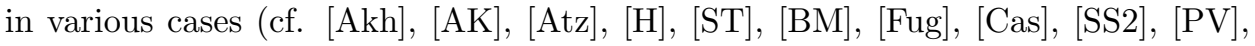
Sch2). Notably, the case when $K$ is a compact semialgebraic set was solved by Schmüdgen [Sch1, and in SS1] Stochel and Szafraniec initiated a study of the case when $K$ is the algebraic variety $\mathcal{Z}_{p}$ induced by a polynomial $p \in \mathbb{R}\left[x_{1}, \ldots, x_{d}\right]$ (cf. [S1], $\mathrm{BiS}$, [PS]). In all of these results, the conditions for $K$-representing measures may be expressed in terms of positivity of the moment matrix and of the localizing matrices directly associated to $\beta^{(\infty)}$. Similarly, in the results of [AK], KN], [CF1], CF7 for the truncated $K$-moment problem, the conditions for $K$-representing measures, including positivity of the moment and localizing matrices, recursiveness, 
and the variety condition, are directly related to the moment data. By contrast, in Theorem 1.4 we see a $K$-moment problem whose solution is not in "closed form", but instead requires parts of the problem to be solved by algorithmic procedures whose outcomes cannot immediately be predicted from the moment data.

Let $p(x, y) \in \mathcal{P}_{n}$. Theorem 1.4 (and its proof) suggest the following question. An affimative answer would reduce the truncated moment problem for $K=\mathcal{Z}_{p}$ to the $p$-pure case.

Question 1.5. Let $p(x, y) \in \mathcal{P}_{n}$ and suppose $M(n)$ is positive, recursively generated, satisfies the variety condition $r \leq v$, and $p(X, Y)=0$ in $\mathcal{C}_{M(n)}$. Does $M(n)$ satisfy at least one of the following properties: $M(2)$ is singular; $M(n)$ is $p$-pure; $M(n)$ has flat data; $M(n)$ is extremal; $M(n)$ is recursively determinate?

We conclude this section with some terminology and background results that we will employ in the sequel. Unless otherwise stated, we are in the general case, i.e., $d \geq 1$. For $p \equiv \sum_{i \in \mathbb{Z}_{+}^{d},|i| \leq n} a_{i} x^{i} \in \mathcal{P}_{n}$, let $\hat{p} \equiv\left(a_{i}\right)$ denote the coefficient vector of $p$ relative to the basis $\mathcal{B}_{n}$ of monomials in $\mathcal{P}_{n}$ in degree-lexicographic order. Following CF2, CF6, we associate to $\beta \equiv \beta^{(2 n)}$ the moment matrix $M(n) \equiv M(n)(\beta)$, with rows and columns $X^{i}$ indexed by the elements of $\mathcal{B}_{n}$. The entry in row $X^{i}$, column $X^{j}$ of $M(n)$ is $\beta_{i+j}\left(i, j \in \mathcal{Z}_{+}^{d},|i|,|j| \leq n\right)$, so $M(n)$ is a real symmetric matrix characterized by $\langle M(n) \hat{p}, \hat{q}\rangle=\Lambda_{\beta}(p q)\left(p, q \in \mathcal{P}_{n}\right)$. If $\mu$ is a representing measure for $\beta$, then $\langle M(n) \hat{p}, \hat{p}\rangle=\Lambda_{\beta}\left(p^{2}\right)=\int p^{2} d \mu \geq 0$, and since $M(n)$ is real symmetric, it follows that $M(n)$ is positive semidefinite $(M(n) \succeq 0)$.

For $p(x) \equiv \sum a_{i} x^{i} \in \mathcal{P}_{n}$, we have the column space element $p(X) \equiv \sum a_{i} X^{i}$, and a calculation shows that $p(X)=M(n) \hat{p}$. If $\beta$ admits a representing measure $\mu$, then

$$
\text { for } p \in \mathcal{P}_{n} \text {, supp } \mu \subseteq \mathcal{Z}_{p} \Longleftrightarrow p(X)=0 \quad \text { (cf. [CF2, Prop. 3.1]). }
$$

It follows from (1.5) that supp $\mu \subseteq \mathcal{V}(M(n))$, whence

$$
r \equiv \operatorname{rank} M(n) \leq \text { card supp } \mu \leq v \equiv \operatorname{card} \mathcal{V}(M(n)) \quad \text { (cf. [CF2, Cor. 3.7]). }
$$

In the sequel we will frequently cite the following basic existence theorem of [CF2], CF6] for a "minimal" representing measure, a representing measure $\mu$ satisfying card supp $\mu=\operatorname{rank} M(n)$.

Theorem 1.6 (Flat Extension Theorem; cf. [CF6, Thms. 1.1-1.2]). $\beta \equiv \beta^{(2 n)}$ has a rank $M(n)$-atomic representing measure if and only if $M(n) \succeq 0$ and $M(n)$ admits a flat moment matrix extension, i.e., a moment matrix extension $M(n+1)$ satisfying rank $M(n+1)=\operatorname{rank} M(n)$. In this case, $\beta^{(2 n+2)}$ admits a unique representing measure, $\mu \equiv \mu_{M(n+1)}$, and $\mu$ satisfies supp $\mu=\mathcal{V}(M(n+1))$ and card supp $\mu=$ rank $M(n)$. Further, $M(n+1)$ admits unique successive positive extensions $M(n+2), M(n+3), \ldots$, and these are flat extensions.

An algebraic proof of Theorem 1.6 was presented by Laurent in $[\mathrm{L}$. Note that for the case of flat data $(M(n) \succeq 0$ and $\operatorname{rank} M(n)=\operatorname{rank} M(n-1))$, Theorem 1.6 (applied to $M(n-1)$ ) implies the existence of a unique (rank $M(n)$-atomic) representing measure for $\beta^{(2 n)}$.

Suppose $M(n)$ is positive and admits a flat extension $M(n+1)$. The unique representing measure for $M(n+1)$ referred to in Theorem 1.6 may be explicitly computed as follows (cf. [CF6, Theorem 1.2]). Let $r=\operatorname{rank} M(n)$, so that 
$\operatorname{card} \mathcal{V}(M(n+1))=r$ and $\mathcal{V}(M(n+1)) \equiv\left\{w_{i}\right\}_{i=1}^{r}$. Let $\mathcal{B} \equiv\left\{X^{i_{1}}, \ldots, X^{i_{r}}\right\}$ denote a basis for $\mathcal{C}_{M(n)}$, and consider the Vandermonde-type matrix

$$
V \equiv V_{\mathcal{B}}:=\left(\begin{array}{ccc}
w_{1}^{i_{1}} & \ldots & w_{r}^{i_{1}} \\
\vdots & & \vdots \\
w_{1}^{i_{r}} & \ldots & w_{r}^{i_{r}}
\end{array}\right) .
$$

Then $V$ is invertible, and CF6 implies that $\beta^{(2 n+2)}$ has the unique representing measure $\mu \equiv \mu_{M(n+1)}$, of the form $\mu=\sum_{i=1}^{r} \rho_{i} \delta_{w_{i}}$, where $\delta_{w_{i}}$ is the unit-mass atomic measure with support $\left\{w_{i}\right\}$, and $\rho \equiv\left(\rho_{1}, \ldots, \rho_{r}\right)$ is determined by $\rho^{t}=$ $V^{-1}\left(\beta_{i_{1}}, \ldots, \beta_{i_{r}}\right)^{t}$. (CF6 shows that $\mu$ is independent of $\mathcal{B}$.)

Suppose $M(n)$ is recursively determinate. In the sequel we require the following result of [F4], which provides an upper bound on the number of extension steps needed to determine whether or not $\beta^{(2 n)}$ has a representing measure.

Theorem 1.7 ([F4, Theorem 4.3]). Suppose $M(n)$ is recursively determinate and $r \equiv \operatorname{rank} M(n) \leq v \equiv \operatorname{card} \mathcal{V}(M(n))<+\infty$. Then $\beta^{(2 n)}$ has a representing measure if and only if there exists $i, 0 \leq i \leq v-r$, such that $M(n)$ admits positive recursively determined extensions $M(n+1), \ldots, M(n+i+1)$, and $M(n+i+1)$ is a flat extension of $M(n+i)$.

We note that the existence or nonexistence of the extensions in Theorem 1.7 can always be determined algorithmically (cf. [F4]). For the $\Gamma$-moment problem, it will only be necessary to apply Theorem 1.7 with $0 \leq i \leq v-r \leq 1$ (cf. Section 5).

We next recall some properties of positive moment matrix extensions that we will refer to in the sequel. A key ingredient in our proofs is a recent result of Bayer and Teichmann $[\mathrm{BT}$, which generalizes the classical theorem of Tchakaloff [T] concerning multivariable cubature (cf. [M], $[\mathrm{P}$, CF5 $)$. The result of Bayer and Teichmann implies that if $\beta \equiv \beta^{(2 n)}$ admits a $K$-representing measure, then $\beta$ admits a finitely atomic $K$-representing measure $\nu$. Since $\nu$ has convergent power moments of all orders, it follows that $M(n)(=M(n)[\nu])$ admits successive positive, recursively generated extensions, namely, $M(n+1)[\nu], M(n+2)[\nu], \ldots$. In the sequel we will show, conversely, that in the $\Gamma$-truncated moment problem, if $Y=X^{3}$ in $M(n)$, then the existence of a positive, recursively generated extension $M(n+2)$ is a sufficient condition for a $\Gamma$-representing measure (cf. Corollary 5.3).

By combining Theorem 1.6 with [BT], we have the following solution of the truncated $K$-moment problem for $K=\mathbb{R}^{d}$, expressed in terms of moment matrix extensions. A generalization to the case when $K$ is a closed semialgebraic subset of $\mathbb{R}^{d}$ appears in [CF6, Cor. 1.4].

Theorem 1.8 (Moment Matrix Extension Theorem; cf. CF3, CF6 ). $\beta^{(2 n)}$ has a representing measure if and only if there is an integer $k \geq 0$ such that $M(n)$ admits a positive moment matrix extension $M(n+k)$ which in turn admits a flat extension $M(n+k+1)$. In this case, we may take $k \leq \operatorname{dim} \mathcal{P}_{2 n}-\operatorname{rank} M(n)$.

Theorem 1.8 is not, by itself, a concrete solution to the truncated $K$-moment problem, but it does provide a framework for obtaining concrete solutions such as Theorem 1.4. In [CF3, Theorem 1.8 was proved for representing measures having convergent moments of all orders, but BT] shows that this restriction in the hypothesis is unnecessary. The upper bound for $k$ comes from BT and improves the bound in CF3. Nevertheless, this bound satisfies $\operatorname{dim} \mathcal{P}_{2 n}-\operatorname{rank} M(n) \geq$ 
$\operatorname{dim} \mathcal{P}_{2 n}-\operatorname{dim} \mathcal{P}_{n}$, and for $d=2$, the latter expression equals $\frac{3}{2} n(n+1)$. By contrast, in the truncated $\Gamma$-moment problem, Corollary 5.3 shows that in Theorem 1.8 , we may take $k \leq 1$.

Consider a real symmetric block matrix $\widetilde{M} \equiv\left(\begin{array}{cc}M & B \\ B^{t} & C\end{array}\right)$. A result of Smul'jan [Smu] implies that $\widetilde{M} \succeq 0$ if and only if $M \succeq 0$, there exists a matrix $W$ such that $B=M W$ (equivalently, $\operatorname{Ran} B \subseteq \operatorname{Ran} M[\mathrm{D}]$ ), and $C \succeq C^{b} \equiv W^{t} M W$ (note that $C^{b}$ is independent of $W$ satisfying $\left.B=M W\right)$. In this case, the matrix $M^{b} \equiv$ $[M ; B]:=\left(\begin{array}{cc}M & B \\ B^{t} & C^{b}\end{array}\right)$ is a positive flat extension of $M$, i.e., rank $M^{b}=\operatorname{rank} M$. Consider a moment matrix extension

$$
M(n+1) \equiv\left(\begin{array}{cc}
M(n) & B(n+1) \\
B(n+1)^{t} & C(n+1)
\end{array}\right) .
$$

If $M(n) \succeq 0$, then $M(n+1)$ is a flat (hence positive) extension of $M(n)$ if and only if $B(n+1)=M(n) W$ (for some $W$ ) and $C(n+1)=C^{b} \equiv W^{t} M(n) W$; equivalently, $M(n+1)=[M(n) ; B(n+1)]$. Suppose $M(n+1) \succeq 0$ and let $p \in \mathcal{P}_{n} ;$ the Extension Principle [F1] shows that if $p(X)=0$ in $\mathcal{C}_{M(n)}$, then $p(X)=0$ in $\mathcal{C}_{M(n+1)}$, i.e., column dependence relations in $M(n)$ extend to $M(n+1)$. It follows that

$$
M(n+1) \succeq 0 \Longrightarrow \mathcal{V}(M(n+1)) \subseteq \mathcal{V}(M(n))
$$

Finally, for the planar case $(d=2)$, we consider the block matrix decomposition $M(n) \equiv(M[i, j])_{0 \leq i, j \leq n}$, where $M[i, j]$ is the matrix with $i+1$ rows and $j+1$ columns of the form

$$
M[i, j] \equiv\left(\begin{array}{ccccc}
\beta_{i+j, 0} & \beta_{i+j-1,1} & \beta_{i+j-2,2} & \ldots & \beta_{i, j} \\
\beta_{i+j-1,1} & \beta_{i+j-2,2} & & \ldots & \beta_{i-1, j+1} \\
\beta_{i+j-2,2} & & & \ldots & \beta_{i-2, j+2} \\
\vdots & & & \ldots & \vdots \\
\beta_{j, i} & \beta_{j-1, i+1} & \beta_{j-2, i+2} & \ldots & \beta_{0, i+j}
\end{array}\right) .
$$

Note that $M[i, j]$ has all of the moments in $\beta^{(2 n)}$ of degree $i+j$ and has the Hankellike property of being constant on cross-diagonals. In particular, in the extension $M(n+1)$, block $C(n+1) \equiv M[n+1, n+1]$ is a Hankel matrix. Note that $B(n+1)=$ $(M[i, n+1])_{0 \leq i \leq n}$. For $0 \leq k \leq n$, we set $[B(n+1)]_{k}=(M[i, n+1])_{0 \leq i \leq k}$. Similarly, for $0 \leq k \leq n$ and $i, j \geq 0, i+j \leq n+1$, we define $\left[X^{i} Y^{j}\right]_{k}:=$ $\left(\beta_{i, j}, \ldots, \beta_{k-j, j}, \ldots, \beta_{i, k-i}\right)^{t}$, the truncation of column $X^{i} Y^{j}$ to rows $X^{r} Y^{s}$ with $r+s \leq k$.

\section{The truncated moment PROBlem With VARIETy $y=x^{3}$}

In this section we prove Theorem 1.1, which will be used to prove Theorem 1.3 in Section 3 and Theorem 1.4-ii) in Section 5. For $d=2$ (the plane), we characterize the existence of representing measures for a positive $\left(y-x^{3}\right)$-pure moment matrix $M(n)$, i.e., a moment matrix $M(n) \succeq 0$ whose column dependence relations are precisely those that can be determined from $Y=X^{3}$ by recursiveness, including all relations of the form

$$
X^{i} Y^{j+1}=X^{i+3} Y^{j}(i, j \geq 0, i+j \leq n-3) .
$$


Our hypothesis implies that $\mathcal{V}(M(n))$ coincides with the curve $\Gamma \equiv\left\{(x, y): y=x^{3}\right\}$ and that rank $M(n)=3 n$, with a basis for $\mathcal{C}_{M(n)}$ of the form

$$
\begin{gathered}
\mathcal{B}=\left\{1, X, Y, X^{2}, X Y, Y^{2}, X^{2} Y, X Y^{2}, Y^{3}, \ldots,\right. \\
\left.X^{2} Y^{i}, X Y^{i+1}, Y^{i+2}, X^{2} Y^{n-2}, X Y^{n-1}, Y^{n}\right\} .
\end{gathered}
$$

(Conversely, it follows from [S1, Prop. 3.4] that if $M(n)$ is any moment matrix for which $\mathcal{V}(M(n))$ coincides with $\Gamma$, then $M(n)$ is $\left(y-x^{3}\right)$-pure.)

As discussed in Section 1, the existence of a representing measure for $\beta^{(2 n)}$ implies that $M(n)$ admits a positive, recursively generated moment matrix extension

$$
M(n+1) \equiv\left(\begin{array}{cc}
M(n) & B(n+1) \\
B(n+1)^{t} & C(n+1)
\end{array}\right) .
$$

We begin by describing concretely the structure of block $B(n+1)$ for such an extension. Positivity of $M(n+1)$ entails $\operatorname{Ran} B(n+1) \subseteq \operatorname{Ran} M(n)$, so we must show that the block that we construct satisfies this range inclusion. Positivity of $M(n+1)$ and the Extension Principle imply that the column relations (2.1) must hold in $\mathcal{C}_{M(n+1)}$. The desired recursiveness of $M(n+1)$ then implies that in $B(n+1)$ we must have column dependence relations of the form

$$
X^{n+1}=X^{n-2} Y, X^{n} Y=X^{n-3} Y^{2}, \ldots, X^{3} Y^{n-2}=Y^{n-1} .
$$

Thus, columns $X^{n+1}, \ldots, X^{3} Y^{n-2}$ in $B(n+1)$ inherit "moment matrix structure" from the column segment $X^{n-2} Y, X^{n-3} Y^{2}, \ldots, X Y^{n-2}, Y^{n-1}$ in $M(n)$. By this we mean that within each block $M[i, n+1](0 \leq i \leq n)$, each cross-diagonal of this segment of columns is constant (cf. (1.9)). Further, we use "old" moments to define $[B(n+1)]_{n-1}$ in columns $X^{2} Y^{n-1}, X Y^{n}, Y^{n+1}$, i.e.,

$$
\left\langleB ( n + 1 ) \left( x^{i} y^{n+1-i} \hat{)},\left(x^{k} y^{l} \widehat{)}\right\rangle=\beta_{i+k, n+1-i+l}(0 \leq i \leq 2, l, k \geq 0, l+k \leq n-1) .\right.\right.
$$

Thus, the segment of columns $X^{2} Y^{n-1}, X Y^{n}, Y^{n+1}$ of $[B(n+1)]_{n-1}$ also displays moment matrix structure. To complete the proof that $[B(n+1)]_{n-1}$ enjoys moment matrix structure, we must establish moment matrix structure within $\left[X^{3} Y^{n-2}\right]_{n-1}$ (defined as in (2.2)) and $\left[X^{2} Y^{n-1}\right]_{n-1}$ (defined in (2.3)).

Lemma 2.1. For $i \geq 1, j \geq 0, i+j \leq n-1,\left\langle B(n+1)\left(x^{3} y^{n-2}\right)^{\uparrow},\left(x^{i-1} y^{j+1}\right)^{\uparrow}\right\rangle$ $=\left\langle B(n+1)\left(x^{2} y^{n-1}\right)^{-},\left(x^{i} y^{j}\right)^{\widehat{\imath}}\right\rangle$.

Proof. We have $\left\langle B(n+1)\left(x^{3} y^{n-2}\right)^{\uparrow},\left(x^{i-1} y^{j+1}\right)^{\uparrow}\right\rangle=\left\langle M(n)\left(y^{n-1}\right)^{\uparrow},\left(x^{i-1} y^{j+1}\right)^{\uparrow}\right\rangle=$ $\beta_{i-1, n+j}$ (by $\left.(2.2)\right)$ and $\left\langle B(n+1)\left(x^{2} y^{n-1}\right)^{\uparrow},\left(x^{i} y^{j}\right)^{\uparrow}\right\rangle=\beta_{i+2, n+j-1}$ (from (2.3)), so it suffices to show that $\beta_{i-1, n+j}=\beta_{i+2, n+j-1}$ for $i, j \geq 0, i+j \leq n-1$. Since $i+j+1 \leq n$, in $M(n)$ we have $\beta_{i-1, n+j}=\left\langle M(n) \widehat{y^{n-2}},\left(x^{i-1} y^{j+2}\right)^{-}\right\rangle=$ $\left\langle M(n)\left(x^{3} y^{n-3}\right) \hat{},\left(x^{i-1} y^{j+2}\right)\right\rangle($ from $(2.2))=\beta_{i+2, n+j-1}$.

We next define the elements of columns $X^{2} Y^{n-1}, X Y^{n}, Y^{n+1}$ in block $B[n, n+1]$ (cf. (2.5) below). To insure moment matrix structure in this block, we propagate the elements of the previously defined column $X^{3} Y^{n-2}$ along the cross diagonals of $B[n, n+1]$, as follows. For $k=0,1,2$ and $i, j \geq 0$ with $i+j=n$ and $0 \leq j \leq n-3+k$, we define

$$
\left\langleB ( n + 1 ) \left( x^{k} y^{n+1-k} \hat{)},\left(x^{i} y^{j}\right) \hat{\rangle}=\left\langleB ( n + 1 ) \left( x^{3} y^{n-2} \hat{)},\left(x^{k+i-3} y^{j-k+3} \hat{)}\right\rangle\right.\right.\right.\right.
$$

$\left(=\left\langle B(n+1) \widehat{y^{n-1}},\left(x^{k+i-3} y^{j-k+3}\right)\right\rangle=\beta_{k+i-3, n+j-k+2}(\right.$ from $\left.(2.2))\right)$. To complete the definition of $B[n, n+1]$, we choose $A, B, C \in \mathbb{R}$ and set $\left\langle B(n+1)\left(x^{2} y^{n-1}\right) \widehat{,} \widehat{y^{n}}\right\rangle=$ 


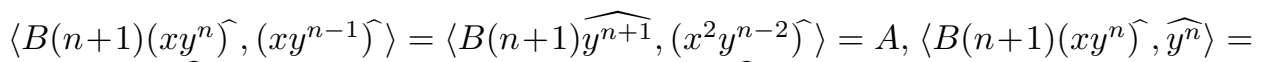
$\left\langle B(n+1) \widehat{y^{n+1}},\left(x y^{n-1}\right)\right\rangle=B$, and $\left\langle B(n+1) \widehat{y^{n+1}}, \widehat{y^{n}}\right\rangle=C$. Thus, $B[n, n+1]$ is of the form

$$
\left(\begin{array}{ccccccccc}
\beta_{2 n+1,0} & \beta_{2 n, 1} & \cdot & \cdot & \cdot & \cdot & . & . & \beta_{n, n+1} \\
\beta_{2 n, 1} & \cdot & \cdot & \cdot & \cdot & \cdot & . & . & \cdot \\
\vdots & \vdots & \vdots & \vdots & \vdots & \vdots & \vdots & \vdots & \vdots \\
\cdot & \cdot & \cdot & \cdot & \cdot & \cdot & \cdot & . & \beta_{3,2 n-2} \\
\cdot & \cdot & \cdot & \cdot & \cdot & \cdot & \cdot & \beta_{3,2 n-2} & A \\
\cdot & \beta_{n+1, n} & \beta_{n, n+1} & \cdot & \cdot & \cdot & \beta_{3,2 n-2} & A & B \\
\beta_{n+1, n} & \beta_{n, n+1} & \cdot & . & . & \beta_{3,2 n-2} & A & B & C
\end{array}\right)
$$

with $\beta_{i, 2 n+1-i}=\beta_{i-3,2 n+2-i} \quad(3 \leq i \leq 2 n+1)$.

Having defined $B(n+1) \equiv B(n+1)_{[A, B, C]}$ to be consistent with recursiveness for $M(n+1)$, we next establish the range inclusion $\operatorname{Ran} B(n+1) \subseteq \operatorname{Ran} M(n)$. It is clear from $(2.2)$ that columns $X^{n+1}, \ldots, X^{3} Y^{n-2}$ of $B(n+1)$ belong to the column space of $M(n)$, which coincides with $\operatorname{Ran} M(n)$, so it suffices to consider columns $X^{2} Y^{n-1}, X Y^{n}$, and $Y^{n+1}$. Let $J$ denote the compression of $M(n)$ to rows and columns indexed by the elements of basis $\mathcal{B}$; thus, $J$ is positive definite $(J \succ 0)$. For $k, l \geq 0$ with $k+l \leq n+1$, let $\widetilde{X^{k} Y^{l}}$ denote the compression of column $X^{k} Y^{l}$ of $(M(n) B(n+1))$ to the rows indexed by the elements of $\mathcal{B}$. Note that the columns of $J$ are of the form $\widehat{X^{p} Y^{q}}$ (in degree-lexicographic order) with $p, q \geq 0$, $p+q \leq n, p \leq 2$. Since $J$ is invertible, for $0 \leq i \leq 2$ and $j=n+1-i$, we may express $\widehat{X^{i} Y^{j}}$ as a linear combination of the columns of $J$, i.e.,

$$
\widetilde{X^{i} Y^{j}}=\sum_{p, q \geq 0, p+q \leq n, p \leq 2} c_{p q}^{(i j)} \widetilde{X^{p} Y^{q}}\left(c_{p q}^{(i j)} \in \mathbb{R}\right) .
$$

We claim that the same relation holds in the columns of $(M(n) \quad B(n+1))$.

Lemma 2.2. In $\left.\mathcal{C}_{(M(n)} B(n+1)\right), X^{i} Y^{j}=\sum_{p, q \geq 0, p+q \leq n, p \leq 2} c_{p q}^{(i j)} X^{p} Y^{q}(0 \leq$ $i \leq 2, j=n+1-i)$.

Proof. In view of (2.6), to prove Lemma 2.2 it suffices to show that for $k, l \geq 0$, $k+l \leq n, k \geq 3$,

$$
\left\langle B(n+1)\left(x^{i} y^{j}\right) \hat{},\left(x^{k} y^{l} \hat{)}\right\rangle=\sum_{p, q \geq 0, p+q \leq n, p \leq 2} c_{p q}^{(i j)}\left\langle M(n)\left(x^{p} y^{q}\right)^{\hat{\gamma}},\left(x^{k} y^{l}\right)^{\widehat{y}}\right\rangle\right.
$$

We note for future reference that (2.6) already shows that

(2.7) holds whenever $k, l \geq 0, k+l \leq n, k \leq 2$.

The proof of (2.7) for $k, l \geq 0, k+l \leq n, k \geq 3$ is by induction on $\rho \equiv k+l \geq 3$. For $\rho=3$, we have $k=3, l=0$, and we will show that in $(M(n) B(n+1))$, row $X^{3}$ equals row $Y$. Since $Y \in \mathcal{B},(2.7)$ holds for $Y(k=0, l=1)$, so it will then follow that (2.7) holds for $X^{3}$, which is the base case of the induction.

In $\mathcal{C}_{M(n)}, Y=X^{3}$, so by self-adjointness, row $X^{3}$ equals row $Y$. (2.2) now implies that row equality also holds in columns $X^{n+1}, \ldots, X^{3} Y^{n-2}$ of $B(n+1)$. To complete the proof of row equality, it suffices to show that for $i=0,1,2$ and $j=n+1-i,\left\langle B(n+1)\left(x^{i} y^{j}\right) \widehat{,}, \widehat{x^{3}}\right\rangle=\left\langle B(n+1)\left(x^{i} y^{j}\right) \widehat{r}, \widehat{y}\right\rangle$. We distinguish 2 cases. 
Case 1: $n=3$. From (2.4), we have $\left\langle B(4)\left(x^{i} y^{j}\right)^{\uparrow}, \widehat{x^{3}}\right\rangle \equiv\left\langle B(4)\left(x^{3} y\right)^{\Upsilon},\left(x^{i} y^{3-i}\right)^{\uparrow}\right\rangle=$ $\left\langle M(3) \widehat{y^{2}},\left(x^{i} y^{3-i}\right)^{\widehat{y}}\right\rangle=\beta_{i, 5-i}=\left\langle B(4)\left(x^{i} y^{4-i}\right), \widehat{y}\right\rangle$ (from $\left.(2.3)\right)$.

Case $2: n \geq 4 .\left\langle B(n+1)\left(x^{i} y^{j}\right)^{-}, \widehat{x^{3}}\right\rangle \equiv \beta_{i+3, n+1-i}($ from $(2.3)$, since $3<n$ )

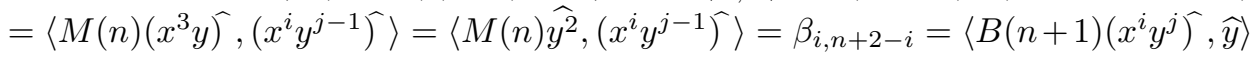
(from (2.3)). This completes the base case $\rho=3$.

Assume now that (2.7) holds whenever $3 \leq \rho<k+l$ (with $k, l \geq 0, k+l \leq n$, $k \geq 3$ ). To establish (2.7) for $k$ and $l$, we first show that for $0 \leq i \leq 2$ and $k, l \geq 0$, with $k+l \leq n$ and $k \geq 3$,

$$
\left\langle B(n+1)\left(x^{i} y^{n+1-i}\right)^{\uparrow},\left(x^{k} y^{l}\right)^{\uparrow}\right\rangle=\beta_{i+k-3, n+l+2-i} .
$$

If $k+l<n$, then $\left\langle B(n+1)\left(x^{i} y^{n+1-i}\right)^{\uparrow},\left(x^{k} y^{l}\right)^{\uparrow}\right\rangle=\beta_{i+k, n+l-i+1}$ (by (2.3)) $=\left\langle M(n)\left(x^{k} y^{l+1}\right)^{\uparrow},\left(x^{i} y^{n-i}\right)^{\uparrow}\right\rangle($ in $M(n)$, since $k+l+1 \leq n)=\left\langle M(n)\left(x^{k-3} y^{l+2}\right)^{\Upsilon}\right.$, $\left.\left(x^{i} y^{n-i}\right)^{r}\right\rangle$ (recursiveness in $\left.M(n)\right)=\beta_{i+k-3, l+n+2-i}$. Next, if $k+l=n$, we have $\left\langle B(n+1)\left(x^{i} y^{n+1-i}\right)^{\uparrow},\left(x^{k} y^{l}\right)^{\uparrow}\right\rangle \equiv\left\langle B(n+1)\left(x^{3} y^{n-2}\right)^{\Upsilon},\left(x^{i+k-3} y^{l+3-i}\right)^{\uparrow}\right\rangle$ (by (2.4), note that $(i+k-3)+(l+3-i)=n)=\left\langle M(n) \widehat{y^{n-1}},\left(x^{i+k-3} y^{l+3-i}\right)^{\uparrow}\right\rangle($ by $(2.2))$ $=\beta_{i+k-3, n+l-i+2}$. The proof of (2.9) is complete. Now, returning to (2.7), we have $\sum_{p, q \geq 0, p+q \leq n, p \leq 2} c_{p q}^{(i j)}\left\langle M(n) \widehat{x^{p} y^{q}}, \widehat{x^{k} y^{l}}\right\rangle=\sum c_{p q}^{(i j)}\left\langle M(n)\left(x^{k} y^{l}\right)^{\uparrow},\left(x^{p} y^{q}\right)^{\widehat{T}}\right\rangle$ (selfadjointness in $M(n))=\sum c_{p q}^{(i j)}\left\langle M(n)\left(x^{k-3} y^{l+1}\right)^{\uparrow},\left(x^{p} y^{q}\right)^{\Upsilon}\right\rangle$ (recursiveness in $M(n)$ ) $=\sum c_{p q}^{(i j)}\left\langle M(n)\left(x^{p} y^{q}\right)^{\Upsilon},\left(x^{k-3} y^{l+1}\right)^{\Upsilon}\right\rangle$ (self-adjointness) $=\left\langle B(n+1)\left(x^{i} y^{n+1-i}\right)^{\Upsilon}\right.$, $\left.\left(x^{k-3} y^{l+1}\right)^{-}\right\rangle$(from (2.7), directly in the case when $k-3 \leq 2$ (cf. (2.8)), or by induction if $k-3 \geq 3$, since $\rho:=(k-3)+(l+1)<k+l)$. Now, $\left\langle B(n+1)\left(x^{i} y^{n+1-i}\right)^{\uparrow},\left(x^{k-3} y^{l+1}\right)^{\uparrow}\right\rangle=\beta_{i+k-3, n+l+2-i}($ from $(2.3)$, since $k+l-2<n)$ $=\left\langle B(n+1)\left(x^{i} y^{n+1-i}\right)^{\uparrow},\left(x^{k} y^{l}\right)^{\uparrow}\right\rangle$ (by (2.9)). This completes the proof of (2.7); hence, the proof of Lemma 2.2 is also complete.

The preceding discussion yields the following result.

Proposition 2.3. If $M(n)$ is positive and recursively generated, with column relations determined entirely from $Y=X^{3}$ by recursiveness, then $M(n)$ admits a moment matrix block $B(n+1) \equiv B(n+1)_{[A, B, C]}$ compatible with a recursively generated extension $M(n+1)$, and any such block $B(n+1)$ satisfies Ran $B(n+1) \subseteq$ Ran $M(n)$.

We note that Proposition 2.3 can be generalized to the case when $M(n)$ is $p$-pure with $p(x, y)=y-x^{k}(k \geq 1)$, resulting in a block $B(n+1) \equiv B(n+1)_{\left[A_{1}, \ldots, A_{k}\right]}$ satisfying $\operatorname{Ran} B(n+1) \subseteq \operatorname{Ran} M(n)$.

For the $\left(y-x^{3}\right)$-pure case, having just described the structure of block $B(n+1)$ for a positive, recursively generated moment matrix extension $M(n+1) \equiv$ $\left(\begin{array}{cc}M(n) & B(n+1) \\ B(n+1)^{t} & C(n+1)\end{array}\right)$, we next consider conditions for the existence of block $C(n+1) \equiv B[n+1, n+1]$ for such an extension. Since $\operatorname{Ran} B(n+1) \subseteq \operatorname{Ran} M(n)$ (Proposition 2.3), there exists a matrix $W$ such that $B(n+1)=M(n) W$. As discussed in Section 1, $M(n+1) \geq 0$ if and only if

$$
C(n+1) \geq C^{b} \equiv B(n+1)^{t} W\left(=W^{t} M(n) W\right) .
$$

Further, $M(n+1)$ is a flat extension of $M(n)$ if and only if $C^{b}$ has the form of a moment matrix block $C(n+1)$ and $C(n+1)=C^{b}$. 
Since $M(n+1)$ is to be positive, the Extension Principle implies that each of the column relations in (2.1) persists in $M(n+1)$. From this and the required recursiveness in $M(n+1)$, it follows that each of the column relations in (2.2) must hold in $M(n+1)$. In particular, these relations must hold in the columns of

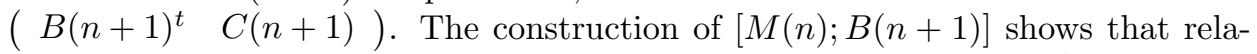
tions (2.2) also hold in $\left(B(n+1)^{t} \quad C^{b}\right)$, so $C(n+1)$ agrees with $C^{b}$ in columns $X^{n+1}, \ldots, X^{3} Y^{n-1}$. Since $C^{b} \equiv\left(C_{i j}^{b}\right)_{1 \leq i, j \leq n+2}$ is positive, hence real symmetric, (2.2) implies that $C^{b}$ has the form of a moment matrix block $C(n+1)$, i.e., $C^{b}$ is Hankel, if and only if

$$
\begin{gathered}
C_{n+1, n-1}^{b}=C_{n, n}^{b}, \\
C_{n+2, n-1}^{b}=C_{n+1, n}^{b},
\end{gathered}
$$

and

$$
C_{n+2, n}^{b}=C_{n+1, n+1}^{b} .
$$

Since the element in row $n$, column $n$ of $C(n+1)$ is $\left\langle C(n+1)\left(x^{2} y^{n-1}\right)^{\uparrow},\left(x^{2} y^{n-1}\right)^{\uparrow}\right\rangle$, the condition $C(n+1) \geq C^{b}$ of $(2.10)$ entails

$$
\left\langle C(n+1)\left(x^{2} y^{n-1}\right)^{\uparrow},\left(x^{2} y^{n-1}\right)^{\hat{\gamma}}\right\rangle \geq C_{n, n}^{b} .
$$

We next compute $C^{b}$ explicitly so as to analyze (2.11)-(2.14). As above, let $J \equiv[M(n)]_{\mathcal{B}}$ denote the compression of $M(n)$ to the rows and columns indexed by basis $\mathcal{B}$, so that $J \succ 0$. Let us write

$$
J \equiv\left(\begin{array}{cc}
M & x \\
x^{t} & \Delta
\end{array}\right)
$$

where $M$ is the compression of $M(n)$ to the rows and columns indexed by the elements of basis $\mathcal{B}$ except for $Y^{n}$ (the final basis element in the degree-lexicographic ordering) and where

$$
\left(\begin{array}{c}
x \\
\Delta
\end{array}\right)
$$

is the compression of column $Y^{n}$ in $M(n)$ to rows indexed by $\mathcal{B}$. Thus, $\Delta=$ $\left\langle M(n) \hat{Y}^{n}, \hat{Y}^{n}\right\rangle=\beta_{0,2 n}$. Since $J \succ 0$, then $M \succ 0$ and $\Delta>x^{t} M^{-1} x$. A calculation shows that

$$
J^{-1}=\left(\begin{array}{cc}
P & v \\
v^{t} & \epsilon
\end{array}\right)
$$

where

$$
P=M^{-1}\left(1+\epsilon x x^{t} M^{-1}\right), v=-\epsilon M^{-1} x, \epsilon=\frac{1}{\Delta-x^{t} M^{-1} x}(>0) .
$$

To compute $C^{\text {b }}$, let $\widetilde{W}=J^{-1}[B(n+1)]_{\mathcal{B}}$, where $[B(n+1)]_{\mathcal{B}}$ is the compression of $B(n+1)$ to the rows indexed by $\mathcal{B}$. Thus, $[M(n)]_{\mathcal{B}} \widetilde{W}=[B(n+1)]_{\mathcal{B}}$. We next define a matrix $W$, with the same number of rows as $M(n)$ and the same number of columns as $\widetilde{W}$. If $X^{i} Y^{j}$ is in basis $\mathcal{B}$, then row $X^{i} Y^{j}$ of $W$ coincides with row $X^{i} Y^{j}$ of $\widetilde{W}$. If $X^{i} Y^{j}$ is not in $\mathcal{B}$, then row $X^{i} Y^{j}$ of $W$ is a row of zeros. Lemma 2.2 implies that $B(n+1)=M(n) W$, and clearly $B(n+1)^{t} W=[B(n+1)]_{\mathcal{B}}^{t} \widetilde{W}$, so (2.10) implies

$$
C^{b}=[B(n+1)]_{\mathcal{B}}^{t} \widetilde{W}
$$


Turning to $(2.14)$, note that $\left[X^{2} Y^{n-1}\right]_{\mathcal{B}}$, the compression of column $X^{2} Y^{n-1}$ in $B(n+1)$ to rows indexed by $\mathcal{B}$, is of the form

$$
\left(\begin{array}{c}
w \\
A
\end{array}\right)
$$

where $w^{t} \equiv\left(w_{1}, \ldots, w_{3 n-1}\right)$ and each $w_{i}$ is an "old" moment of the form $\beta_{i j}$ with $i, j \geq 0$ and $i+j \leq 2 n$. Let $r_{1}, \ldots, r_{3 n-1}$ denote the successive row vectors of $P$ and let $v^{t}=\left(v_{1}, \ldots, v_{3 n-1}\right)$. Then $J^{-1}\left[X^{2} Y^{n-1}\right]_{\mathcal{B}} \equiv\left(c_{1}(A), \ldots, c_{3 n}(A)\right)^{t}$, where

$$
c_{i}(A) \equiv\left\langle r_{i}, w\right\rangle+v_{i} A(1 \leq i \leq 3 n-1)
$$

and

$$
c_{3 n}(A) \equiv\langle v, w\rangle+\epsilon A
$$

Now, $C_{n, n}^{b}=\left\langle C^{b}\left(x^{2} y^{n-1}\right) \hat{)},\left(x^{2} y^{n-1}\right) \hat{\rangle}=\left[X^{2} Y^{n-1}\right]_{\mathcal{B}}^{t} J^{-1}\left[X^{2} Y^{n-1}\right]_{\mathcal{B}}\right.$ (by (2.15)) $=\left(\begin{array}{ll}w^{t} & A\end{array}\right)\left(\begin{array}{c}c_{1}(A) \\ \vdots \\ c_{3 n}(A)\end{array}\right)=w_{1} c_{1}(A)+\cdots+w_{3 n-1} c_{3 n-1}(A)+A(\langle v, w\rangle+\epsilon A)$ $=\epsilon A^{2}+2\langle v, w\rangle A+\omega$, where $\omega:=\left\langle r_{1}, w\right\rangle w_{1}+\cdots+\left\langle r_{3 n-1}, w\right\rangle w_{3 n-1}=\langle P w, w\rangle$. Setting $f(A)=\epsilon A^{2}+2\langle v, w\rangle A+\omega$, we have

$$
C_{n, n}^{b}=f(A) \text {. }
$$

Returning to (2.14), moment matrix structure requires $\left\langle C(n+1)\left(x^{2} y^{n-1}\right)^{\widehat{V}},\left(x^{2} y^{n-1}\right)^{\widehat{\gamma}}\right\rangle$ $=\left\langle C(n+1)\left(x^{3} y^{n-2}\right)^{-},\left(x y^{n}\right)^{\hat{\gamma}}\right\rangle=\left\langle M(n+1) \widehat{y^{n-1}},\left(x y^{n} \hat{)}\right\rangle\right.$ (by recursiveness in $M(n+1))=\beta_{1,2 n-1}$. Now (2.14) may be expressed as

$$
\beta_{1,2 n-1} \geq f(A):=\epsilon A^{2}+2\langle v, w\rangle A+\omega .
$$

The minimum value of $f(A)$ occurs with

$$
A=A_{\min }:=\frac{-\langle v, w\rangle}{\epsilon}
$$

and

$$
f\left(A_{\min }\right)=\psi(\beta):=\frac{\omega \epsilon-\langle v, w\rangle^{2}}{\epsilon} .
$$

Note that $\psi(\beta)$ is a rational expression in the original moment data $\beta^{(2 n)}$.

The main result of this section is the following reformulation of Theorem 1.1. For $n \geq 3$, this result characterizes the existence of representing measures in the truncated moment problem when the variety of $M(n)$ coincides with $y=x^{3}$.

Theorem 2.4. Let $n \geq 3$. Suppose $M(n)$ is positive and $\left(y-x^{3}\right)$-pure; i.e., $\mathcal{V}(M(n))$ is completely determined (via recursiveness) by the column relation $Y=$ $X^{3}$. The following are equivalent for $\beta \equiv \beta^{(2 n)}$ :

i) $\beta$ has a representing measure (necessarily supported in $y=x^{3}$ );

ii) $M(n)$ has a flat extension $M(n+1)$ (and $\beta$ has a corresponding $3 n$-atomic minimal representing measure supported in $\left.y=x^{3}\right)$;

iii) $\beta_{1,2 n-1}>\psi(\beta)$;

iv) $M(n)$ admits a positive, recursively generated extension $M(n+1)$. 
Proof. We will prove $i) \Longrightarrow i v) \Longrightarrow i i i) \Longrightarrow i i$ ), and $i i) \Longrightarrow i$ ) follows from Theorem 1.6. As discussed in the Introduction, the result of Bayer and Teichmann BT implies that if $\beta$ has a representing measure, then $\beta$ admits a finitely atomic representing measure $\nu$. Since $\nu$ has convergent moments of all orders, $M(n+1) \equiv$ $M(n+1)[\nu]$ is a moment matrix extension of $M(n)$ which itself has a representing measure (namely, $\nu$ ), so $M(n+1)$ is a positive, recursively generated extension of $M(n)$; thus $i) \Longrightarrow i v)$.

For $i v) \Longrightarrow i i i)$, assume that $M(n+1)$ is a positive, recursively generated moment matrix extension of $M(n)$, with $B(n+1) \equiv B(n+1)_{[A, B, C]}$ as described at the beginning of this section. Let $M^{b} \equiv[M(n) ; B(n+1)]$ denote the corresponding flat extension (positive, but not necessarily a moment matrix), and let $C^{b}$ denote the $(n+2) \times(n+2)$ lower right-hand block of $M^{b}$, with rows and columns indexed as $X^{n+1}, X^{n} Y, \ldots, Y^{n+1}$. Since $M(n+1)$ is positive and recursively generated, from (2.14), (2.19), and (2.21) we have $\beta_{1,2 n-1} \geq \psi(\beta)$, and we claim that $\beta_{1,2 n-1}>$ $\psi(\beta)$. Suppose to the contrary that $\beta_{1,2 n-1}=\psi(\beta)$. (2.19) then implies that $\beta_{1,2 n-1}=\psi(\beta)=f\left(A_{\min }\right) \leq f(A) \leq \beta_{1,2 n-1}$. Since $A=A_{\min }$ is the unique minimum point for $f(A)$, it follows that in $B(n+1)$ we have $A=A_{\min }$. We will show that for an appropriate choice of $B$, say $B=B_{0}$, and for any $C$, the flat extension $\left[M(n) ; B(n+1)_{\left[A_{\min }, B_{0}, C\right]}\right]$ is a moment matrix, and this will lead to a contradiction.

Since $M(n+1)$ is recursively generated, $M(n+1)$ coincides with $M^{b}$ in columns $X^{n+1}, \ldots, X^{3} Y^{n-2}$. Our immediate goal is to show that $M(n+1)$ and $M^{b}$ also coincide in column $X^{2} Y^{n-1}$. Recall from Proposition 2.3 that in $\left.\mathcal{C}_{(} M(n) \quad B(n+1)\right)$ ' $X^{2} Y^{n-1}$ may be expressed as a linear combination of the basis elements $p_{1}(X, Y)$, $\ldots, p_{3 n}(X, Y)$, where $p_{1}, \ldots, p_{3 n}$ is a listing of the monomials corresponding to basis $\mathcal{B}$ in degree-lexicographic order; in particular, $p_{3 n}(x, y)=y^{n}$. From $J^{-1}\left[X^{2} Y^{n-1}\right]_{\mathcal{B}}$ $\equiv\left(c_{1}(A), \ldots, c_{3 n}(A)\right)^{t}$ (cf. (2.16)-(2.17)), we may express this relation as

$$
\begin{array}{r}
X^{2} Y^{n-1}=c_{1}\left(A_{\min }\right) p_{1}(X, Y)+\cdots \\
+c_{3 n-1}\left(A_{\min }\right) p_{3 n-1}(X, Y)+c_{3 n}\left(A_{\min }\right) p_{3 n}(X, Y) .
\end{array}
$$

By the flat extension construction (cf. Section 1), the same relation holds in $\mathcal{C}_{M^{\mathrm{b}}}$. We claim that (2.22) also holds in $\mathcal{C}_{M(n+1)}$. Let $\widetilde{C}(n+1)$ denote the compression of $M(n+1)$ to the rows and columns indexed by $X^{n+1}, \ldots, X^{3} Y^{n-2}, X^{2} Y^{n-1}$, and let $\widetilde{C^{b}}$ denote the corresponding compression of $M^{b}$. Since $\widetilde{C^{b}}$ coincides with $\widetilde{C}(n+1)$ in columns $X^{n+1}, \ldots, X^{3} Y^{n-2}$ and $\widetilde{C^{b}}$ and $\widetilde{C}(n+1)$ are real symmetric, it follows that $\widetilde{C^{b}}$ coincides with $\widetilde{C}(n+1)$ except possibly in the lower right corner position, corresponding to row and column $X^{2} Y^{n-1}$. We claim that $\widetilde{C^{b}}$ and $\widetilde{C}(n+1)$ agree in this position as well. Indeed, $\beta_{1,2 n-1}=\left\langle B(n+1)\left(x y^{n}\right)^{-}, \widehat{y^{n-1}}\right\rangle$ (by $\left.(2.3)\right)$ $=\left\langle M(n+1)\left(x y^{n}\right)^{\uparrow}, \widehat{y^{n-1}}\right\rangle=\left\langle M(n+1) \widehat{y^{n-1}},\left(x y^{n}\right)^{\uparrow}\right\rangle$ (since $M(n+1)$ $\left.=M(n+1)^{t}\right)=\left\langle M(n+1)\left(x^{3} y^{n-2}\right)^{-},\left(x y^{n}\right)^{-}\right\rangle$(by recursiveness in $\left.M(n+1)\right)$ $=\left\langle C(n+1)\left(x^{2} y^{n-1}\right)^{\Upsilon},\left(x^{2} y^{n-1}\right)^{\Upsilon}\right\rangle($ since $C(n+1)$ is Hankel $) \geq C_{n, n}^{b}$ (by (2.14)) $=f\left(A_{\min }\right)=\beta_{1,2 n-1}$ (by (2.18), since $A=A_{\text {min }}$ and $\left.f\left(A_{\text {min }}\right)=\beta_{1,2 n-1}\right)$. Thus, $\left\langle C(n+1)\left(x^{2} y^{n-1}\right)^{\uparrow},\left(x^{2} y^{n-1}\right)^{\Upsilon}\right\rangle=C_{n, n}^{b}$, whence $\widetilde{C}(n+1)=\widetilde{C}^{b}$. Now let $\widetilde{M}(n+1)$ denote the compression of $M(n+1)$ to rows and columns indexed by the monomials of $\mathcal{P}_{n}$ and by $X^{n+1}, \ldots, X^{3} Y^{n-2}, X^{2} Y^{n-1}$. Let $\widetilde{M^{b}}$ denote the corresponding 
compression of $M^{b}$. We have just shown that $\widetilde{M}(n+1)=\widetilde{M^{b}}$. In $\mathcal{C}_{\widetilde{M^{b}}}$, relation (2.22) holds, so the same relation holds in $\mathcal{C}_{\widetilde{M}(n+1)}$. Since $M(n+1)$ is positive, it now follows from the Extension Principle that (2.22) holds in $\mathcal{C}_{M(n+1)}$, whence $M(n+1)$ and $M^{b}$ coincide in column $X^{2} Y^{n-1}$. In particular, since the Hankel matrix $C(n+1)$ coincides with $C^{b}$ in columns $X^{3} Y^{n-2}$ and $X^{2} Y^{n-1}$, it follows that (2.11) and (2.12) hold in $C^{b}$, a fact we will use later.

We next analyze the elements in row $X Y^{n}$, column $X Y^{n}$ of $C(n+1)$ and $C^{b}$. Denote $\left[X Y^{n}\right]_{\mathcal{B}}$ in $B(n+1)$ by

$$
\left(\begin{array}{c}
u \\
B
\end{array}\right)
$$

where $u^{t} \equiv\left(u_{1}, \ldots, u_{3 n-1}\right), u_{1}, \ldots, u_{3 n-2}$ are "old" moments and $u_{3 n-1}=A_{\min }$. Thus, $J^{-1}\left[X Y^{n}\right]_{\mathcal{B}} \equiv\left(d_{1}(B), \ldots, d_{3 n}(B)\right)$, where $d_{i}(B)=\left\langle r_{i}, u\right\rangle+v_{i} B(1 \leq i \leq 3 n-$ 1) and $d_{3 n}(B)=\langle v, u\rangle+\epsilon B$. From $(2.15)$, we have $C_{n+1, n+1}^{b}=\left\langle C^{b}\left(x y^{n}\right)^{\uparrow},\left(x y^{n}\right)^{\widehat{\gamma}}\right\rangle=$ $\left[X Y^{n}\right]_{\mathcal{B}}^{t} J^{-1}\left[X Y^{n}\right]_{\mathcal{B}}=u_{1} d_{1}(B)+\cdots+u_{3 n-1} d_{3 n-1}(B)+B d_{3 n}(B)$, whence

$$
C_{n+1, n+1}^{b}=\rho(B):=\epsilon B^{2}+2 B\langle u, v\rangle+\gamma,
$$

with $\gamma:=\langle P u, u\rangle=u_{1}\left\langle r_{1}, u\right\rangle+\cdots+u_{3 n-1}\left\langle r_{3 n-1}, u\right\rangle$. Since $M(n+1)$ is positive, we have

$$
\left\langle C(n+1)\left(x y^{n}\right)^{\widehat{T}},\left(x y^{n}\right)^{\widehat{\gamma}}\right\rangle \geq C_{n+1, n+1}^{b}=\rho(B) .
$$

Next, observe that $\left\langle C(n+1)\left(x y^{n}\right)^{\uparrow},\left(x y^{n}\right)^{\uparrow}\right\rangle=\left\langle C(n+1)\left(x^{2} y^{n-1}\right)^{\uparrow}, \widehat{y^{n+1}}\right\rangle$, and recall from above that $\left\langle C(n+1)\left(x^{2} y^{n-1}\right)^{-}, \widehat{y^{n+1}}\right\rangle$ coincides with $\left\langle C^{b}\left(x^{2} y^{n-1}\right)^{\widehat{y}}, \widehat{y^{n+1}}\right\rangle$. To compute this, denote $\left[Y^{n+1}\right]_{\mathcal{B}}$ in $B(n+1)$ by

$$
\left(\begin{array}{c}
y \\
A_{\min } \\
B \\
C
\end{array}\right),
$$

where $y^{t}:=\left(y_{1}, \ldots, y_{3 n-3}\right)$ and each $y_{i}$ is an "old" moment. Now, since $M(n+1)$ and $M^{b}$ agree in column $X^{2} Y^{n-1}$, it follows from (2.15) that

$$
\begin{aligned}
\left\langle C(n+1)\left(x^{2} y^{n-1}\right), \widehat{y^{n+1}}\right\rangle= & \left\langle C^{b}\left(x^{2} y^{n-1} \widehat{)}, \widehat{y^{n+1}}\right\rangle=\left[Y^{n+1}\right]_{\mathcal{B}}^{t} J^{-1}\left[X^{2} Y^{n-1}\right]_{\mathcal{B}}\right. \\
= & y_{1} c_{1}\left(A_{\min }\right)+\cdots+y_{3 n-3} c_{3 n-3}\left(A_{\min }\right) \\
& +A_{\min } c_{3 n-2}\left(A_{\min }\right)+B c_{3 n-1}\left(A_{\min }\right)+C c_{3 n}\left(A_{\min }\right) .
\end{aligned}
$$

Since $A=A_{\text {min }}$, it follows from (2.17) and (2.20) that $c_{3 n}\left(A_{\min }\right)=0$, whence $\left\langle C^{b}\left(x^{2} y^{n-1}\right)^{-}, \widehat{y^{n+1}}\right\rangle$ may be expressed as $\left\langle C^{b}\left(x^{2} y^{n-1}\right)^{-} \widehat{y^{n+1}}\right\rangle \equiv \delta(B):=\tau+\lambda B$, where $\tau$ and $\lambda$ depend on $\beta$ and $A_{\text {min }}$, but are independent of $B$ and $C$. Now, using (2.24), we have $\delta(B)=\left\langle C^{b}\left(x^{2} y^{n-1}\right) \widehat{y^{n+1}}\right\rangle=\left\langle C(n+1)\left(x^{2} y^{n-1}\right)^{\widehat{y}}, \widehat{y^{n+1}}\right\rangle=$ $\left\langle C(n+1)\left(x y^{n}\right)^{-},\left(x y^{n}\right)^{-}\right\rangle \geq C_{n+1, n+1}^{b}=\rho(B)$. Let $h(b):=\rho(b)-\delta(b) \equiv \epsilon b^{2}+\kappa b+\alpha$ (where $\kappa$ and $\alpha$ depend only on moment data $\beta$ ). Since $h(B) \leq 0$ and $\epsilon>0$, there exists $b \equiv B_{0}$ such that $h\left(B_{0}\right)=0$. Consider $B(n+1) \equiv B(n+1)_{\left[A_{\text {min }}, B_{0}, C\right]}$ and the corresponding flat extension $M^{b} \equiv\left[M(n) ; B(n+1)_{\left[A_{m i n}, B_{0}, C\right]}\right]$. We claim that $M^{b}$ is a moment matrix. We have already shown that (2.11) and (2.12) hold in any flat extension of the form $\left[M(n) ; B(n+1)_{\left[A_{m i n}, B, C\right]}\right]$. The preceding argument shows that $\delta\left(B_{0}\right)=\left\langle C^{b}\left(x^{2} y^{n-1}\right)^{\Upsilon},\left(y^{n+1}\right)^{\uparrow}\right\rangle=\left\langle C(n+1)\left(x^{2} y^{n-1}\right)^{\uparrow},\left(y^{n+1}\right)^{\Upsilon}\right\rangle$ $=\left\langle C(n+1)\left(x y^{n}\right)^{\uparrow},\left(x y^{n}\right)^{-}\right\rangle \geq C_{n+1, n+1}^{b}=\rho\left(B_{0}\right)=\delta\left(B_{0}\right)$, whence $C_{n+2, n}^{b}=$ 
$\left\langle C^{b}\left(x^{2} y^{n-1}\right)^{\uparrow},\left(y^{n+1}\right)^{\uparrow}\right\rangle=C_{n+1, n+1}^{b}$. Thus, (2.13) also holds, whence $M^{\mathrm{b}}$ is a moment matrix. This completes the proof that if $M(n)$ admits a positive, recursively generated extension with $\beta_{1,2 n-1}=\psi(\beta)$, then $M(n)$ admits a moment matrix flat extension $\left[M(n) ; B(n+1)_{\left[A_{m i n}, B_{0}, C\right]}\right]$.

To obtain a contradiction, let us redefine $M(n+1)$ as

$$
M(n+1):=\left[M(n) ; B(n+1)_{\left[A_{m i n}, B_{0}, C\right]}\right] .
$$

It follows from Theorem 1.6 that $M(n+1)$ admits a representing measure, whence (1.6) implies rank $M(n+1) \leq \operatorname{card} \mathcal{V}(M(n+1))$. Consider $g(x, y)=$ $x^{2} y^{n-1}-\left(c_{1}\left(A_{\min }\right) p_{1}(x, y)+\cdots+c_{3 n-1}\left(A_{\min }\right) p_{3 n-1}(x, y)\right)$. Since $c_{3 n}\left(A_{\min }\right)=$ 0 , (2.22) implies $g(X, Y)=0$ in $\mathcal{C}_{M(n+1)}$. Since $Y=X^{3}$ in $\mathcal{C}_{M(n+1)}$, we have $\mathcal{V}(M(n+1)) \subseteq\left\{\left(x, x^{3}\right): g\left(x, x^{3}\right)=0\right\}$. With $y=x^{3}, G(x):=g\left(x, x^{3}\right)$ satisfies $\operatorname{deg} G=3 n-1\left(\right.$ since $x^{2} y^{n-1}=x^{3 n-1}$ and $\left.p_{3 n-1}(x, y)=x y^{n-1}=x^{3 n-2}\right)$. The preceding degree calculation implies that $\operatorname{card} \mathcal{V}(M(n+1)) \leq 3 n-1<3 n=$ rank $M(n)=\operatorname{rank} M(n+1)$, a contradiction to the existence of a representing measure for $M(n+1)$. This completes the proof that if $\beta_{1,2 n-1}=\psi(\beta)$, then $M(n)$ admits no positive, recursively generated extension $M(n+1)$. Thus, the proof of $i v) \Longrightarrow i i i)$ is also complete.

To complete the proof of Theorem 2.4, we will show $i i i) \Longrightarrow i i)$, i.e., if $\beta_{1,2 n-1}>$ $\psi(\beta)$, then $M(n)$ admits a flat extension $M(n+1)$, which then implies the existence of a rank $M(n)$-atomic (minimal) representing measure, necessarily supported in $y=x^{3}$ (cf. (1.5)). A flat extension requires choices of $A, B, C$ such that $M^{b} \equiv$ $\left[M(n) ; B(n+1)_{[A, B, C]}\right]$ satisfies (2.11)-(2.13). For (2.11), which entails $C_{n+1, n-1}^{b}=$ $C_{n, n}^{b}$, recall that $C_{n, n}^{b}=f(A)$ (cf. (2.18)). Also, from (2.2), we have $X^{3} Y^{n-2}=$ $Y^{n-1}$ in $\mathcal{C}_{M^{b}}$, so $C_{n+1, n-1}^{b}=\left\langle C^{b}\left(x^{3} y^{n-2}\right)^{-},\left(x y^{n}\right)^{\widehat{\gamma}}\right\rangle=\left\langle C^{b} \widehat{y^{n-1}},\left(x y^{n}\right)^{\widehat{ }}\right\rangle=\beta_{1,2 n-1}$. Thus, (2.11) reduces to solving $\beta_{1,2 n-1}=f(A)$. Since $\beta_{1,2 n-1}>\psi(\beta)\left(=f\left(A_{\text {min }}\right)\right)$, then there exist distinct values $A_{1}, A_{2}$, such that $f\left(A_{i}\right)=\beta_{1,2 n-1}(i=1,2)$, so (2.11) holds if we use either $A=A_{1}$ or $A=A_{2}$ in $B(n+1)$. Since, from (2.17), $c_{3 n}\left(A_{i}\right)=\langle v, w\rangle+\epsilon A_{i}$, we may choose $i$ so that $c \equiv c_{3 n}\left(A_{i}\right) \neq 0$. With this choice, $A=A_{i}$, we will show that there exist $B, C$ such that the flat extension $\left[M(n) ; B(n+1)_{[A, B, C]}\right]$ is a moment matrix.

Since (2.11) already holds, we consider (2.12) and (2.13). For (2.12), we require $C_{n+1, n}^{b}=C_{n+2, n-1}^{b}$. Now, $C_{n+2, n-1}^{b}=\left\langle M^{b}\left(x^{3} y^{n-2}\right)^{-}, \widehat{y^{n+1}}\right\rangle=\left\langle M^{b} \widehat{y^{n-1}}, \widehat{y^{n+1}}\right\rangle$ (since $(2.2)$ holds in $\left.M^{\mathrm{b}}\right)=\left\langle M^{\mathrm{b}} \widehat{y^{n+1}}, \widehat{y^{n-1}}\right\rangle$ (since $M^{\mathrm{b}}$ is real symmetric) $=\left\langle B(n+1) \widehat{y^{n+1}}, \widehat{y^{n-1}}\right\rangle=\beta_{0,2 n}$ (from (2.3)). Thus, (2.12) is equivalent to

$$
C_{n+1, n}^{b}=\beta_{0,2 n} \text {. }
$$

From (2.15), we have $C_{n+1, n}^{b}=\left[X Y^{n}\right]_{\mathcal{B}}^{t} J^{-1}\left[X^{2} Y^{n-1}\right]_{\mathcal{B}}$, where $J^{-1}\left[X^{2} Y^{n-1}\right]_{\mathcal{B}}$ $\equiv\left(c_{1}(A), \ldots, c_{3 n}(A)\right)^{t}$ and $\left[X Y^{n}\right]_{\mathcal{B}}^{t} \equiv\left(q_{1}, \ldots, q_{3 n-2}, A, B\right)$, with each $q_{i}$ an "old" moment. Thus, $C_{n+1, n}^{b}=q_{1} c_{1}(A)+\cdots+q_{3 n-2} c_{3 n-2}(A)+A c_{3 n-1}(A)+B c_{3 n}(A)$. Since $c_{3 n}(A) \neq 0$, there exists a unique $B$ such that $(2.25)$ holds. It remains to choose $C$ for block $B(n+1)$ such that $(2.13)$ holds, i.e., $C_{n+1, n+1}^{b}=C_{n+2, n}^{b}$. Using (2.15), $C_{n+1, n+1}^{b}=\left\langle C^{b}\left(x y^{n}\right)^{\uparrow},\left(x y^{n}\right)^{\uparrow}\right\rangle=\left[X Y^{n}\right]_{\mathcal{B}}^{t} J^{-1}\left[X Y^{n}\right]_{\mathcal{B}}$, and since $\left[X Y^{n}\right]_{\mathcal{B}}$ is independent of $C$, so is $C_{n+1, n+1}^{b}$ (though it depends on $A$ and $B$ ). Further, from (2.15) we see that $C_{n+2, n}^{b}=\left[Y^{n+1}\right]_{\mathcal{B}}^{t} J^{-1}\left[X^{2} Y^{n-1}\right]_{\mathcal{B}}$, where $\left[Y^{n+1}\right]_{\mathcal{B}}^{t} \equiv$ $\left(y_{1}, \ldots, y_{3 n-3}, A, B, C\right)$ and each $y_{i}$ is an "old" moment. Thus, $C_{n+2, n}^{b}=y_{1} c_{1}(A)+$ $\cdots+y_{3 n-3} c_{3 n-3}(A)+A c_{3 n-2}(A)+B c_{3 n-1}(A)+C c_{3 n}(A)$. Since $c_{3 n}(A) \neq 0$, it 
follows that there is a unique $C$ such that (2.13) holds. Thus, $M(n)$ has the flat extension $M(n+1)_{[A, B, C]}$; the proof is complete.

Remark 2.5. Note that in the proof of Theorem 2.4, the number of conditions for a flat extension (cf. (2.11)-(2.13)) matches the number of free choices in constructing $B(n+1) \equiv B(n+1)_{[A, B, C]}$. For curves of higher degree, such as $y=x^{k}$, the number of conditions for a flat extension exceeds the number of free choices in constructing $B(n+1)$. For $k=4$, there are four free choices in $B(n+1)$ and six constraints for a flat extension. We therefore cannot expect that, for $k>3$, representing measures in the pure case will always correspond to flat extensions; rather, we surmise that representing measures in the pure case will sometimes entail several rank-increasing extensions, followed by a flat extension. Similarly, we would expect condition iii) of Theorem 2.4 to be replaced by a set of several conditions.

\section{A CONSISTENT SEQUENCE HAVING NO REPRESENTING MEASURE}

In this section we provide an example illustrating Theorem 2.4 (equivalently, Theorem 1.1). As a special case, we describe a moment matrix $M(3)$ that is positive and consistent, with $Y=X^{3}$ and rank $M(3)<\operatorname{card} \mathcal{V}(M(n))$, but for which $\beta^{(6)}$ admits no representing measure. This provides a negative answer to CFM, Question 1.1] and proves Theorem 1.3.

Lemma 3.1. $\beta \equiv \beta^{(2 n)}$ is consistent if $M(n)$ is $p$-pure, where deg $p(x, y)=n, p$ is irreducible in $\mathbb{R}[x, y]$ and $\mathcal{Z}_{p}$ is infinite.

Proof. The hypothesis implies that $\mathcal{V} \equiv \mathcal{V}(M(n))=\mathcal{Z}_{p}$. Suppose $r(x, y) \in \mathcal{P}_{2 n}$ with $r \mid \mathcal{V} \equiv 0$. Since $p$ is irreducible and $\mathcal{Z}_{p}$ is infinite, it follows from [S1, Prop. 3.4] that there exists $q(x, y) \in \mathbb{R}[x, y]$ such that $r=p q$, and thus $\operatorname{deg} q=\operatorname{deg} r-\operatorname{deg} p \leq$ $n$. Now, $M(n) \widehat{p}=p(X, Y)=0$, so $\Lambda_{\beta}(r)=\langle M(n) \widehat{p}, \widehat{q}\rangle=0$, and it follows that $\beta$ is consistent.

Example 3.2. Consider $M(3)$ defined by

$$
M(3)=\left(\begin{array}{cccccccccc}
1 & 0 & 0 & 1 & 2 & 5 & 0 & 0 & 0 & x \\
0 & 1 & 2 & 0 & 0 & 0 & 2 & 5 & 14 & 42 \\
0 & 2 & 5 & 0 & 0 & x & 5 & 14 & 42 & 132 \\
1 & 0 & 0 & 2 & 5 & 14 & 0 & 0 & x & 0 \\
2 & 0 & 0 & 5 & 14 & 42 & 0 & x & 0 & 0 \\
5 & 0 & x & 14 & 42 & 132 & x & 0 & 0 & 0 \\
0 & 2 & 5 & 0 & 0 & x & 5 & 14 & 42 & 132 \\
0 & 5 & 14 & 0 & x & 0 & 14 & 42 & 132 & r \\
0 & 14 & 42 & x & 0 & 0 & 42 & 132 & r & s \\
x & 42 & 132 & 0 & 0 & 0 & 132 & r & s & t
\end{array}\right) .
$$

It is clear that $Y=X^{3}$ in $\mathcal{C}_{M(3)}$. Let $x_{1}=\frac{1}{2}(\sqrt{85}-9) \approx 0.109772$. A straightforward calculation with nested determinants shows that if $0 \leq x<x_{1}$, then $M(2) \succ 0$ and $M_{8}$, the compression of $M(3)$ to the first 8 rows and columns, is positive with $\operatorname{rank} M_{8}=7$.

We first consider the case when $x=0, r=429, s=1422$, and $t=4798$. A further calculation with nested determinants implies that $M(3) \succeq 0$ with $\operatorname{rank} M(3)=9$, whence $\mathcal{V}(M(3))=\Gamma \equiv\left\{(x, y): y=x^{3}\right\}$. Thus $9=\operatorname{rank} M(3)<\operatorname{card} \mathcal{V}(M(3))=$ $+\infty$, and Lemma 3.1 implies that $M(3)$ is consistent. We claim that $\beta^{(6)}$ admits no 
representing measure. Indeed, a calculation shows that $\psi(\beta)=1429>1422=\beta_{1,5}$, so the result follows from Theorem 2.4. In more detail, following the proof of Theorem 2.4, we see that block $\widetilde{C}(4)$ in any recursively generated moment matrix extension $M(4)$ of $M(3)$ must be of the form

$$
\widetilde{C}(4)=\left(\begin{array}{ccc}
14 & 42 & 132 \\
42 & 132 & 429 \\
132 & 429 & 1422
\end{array}\right) .
$$

Now, det $\widetilde{C}(4)=-341$, so $M(4)$ cannot be positive. Since $M(3)$ admits no positive recursively generated extension, it follows from [BT] (as in the proof of Theorem 2.4 ) that $\beta^{(6)}$ admits no representing measure. This example proves Theorem 1.3.

We next consider the case of (3.1) when $x=\frac{1}{10}, r=600, s=\frac{526337068574699}{741609900} \approx$ 709722 , and $t=\frac{2849859462886367177063452994221}{251774383728200998950} \approx 11319100143$. As in the previous case, $M(3) \succeq 0$, with rank $M(3)=9$, whence $\mathcal{V}(M(3))=\Gamma$ and rank $M(3)<$ card $\mathcal{V}(M(3))$. Lemma 3.1 implies that $M(3)$ is consistent. A calculation shows that $\psi(\beta)=\frac{526337068574699}{741609900}=\beta_{1,5}$, so Theorem 2.4 again implies that $\beta^{(6)}$ has no representing measure.

For our final case of (3.1), we again set $x=\frac{1}{10}$ and $r=600$. A calculation shows that in this case $\psi(\beta)$ is independent of $s$ and $t$, with $\psi(\beta)=\frac{526337068574699}{741609900}$ (as above). We set $s=\psi(\beta)+1$. Let $t_{0}$ denote the unique value for $\beta_{0,6}$ such that $\operatorname{rank} M(3)=8$, and set $t=t_{0}+1$. Now, $M(3)$ is positive with rank 9 , so $\mathcal{V}(M(3))=\Gamma$ and Theorem 2.4 applies. Since $s>\psi(\beta)$, there exists a flat extension $M(4)$ and a corresponding 9-atomic representing measure supported in $y=x^{3}$. To find the atoms, we construct $B(4)$ as in the proof of Theorem 2.4. For A, we choose the smaller of the two roots of $C_{3,3}^{b}(A)=\beta_{1,5}(=s)$, and then $B$ and $C$ are uniquely determined. In accordance with Lemma 2.2 and Proposition 2.3 , we next express each column $X^{i} Y^{j}$ of $B(4)$ as a linear combination of columns of $M(3)$, i.e., $X^{i} Y^{j}=p_{i j}(X, Y)$ for some $p_{i j} \in \mathcal{P}_{3}(i, j \geq 0, i+j=4)$. Setting $q_{i j}=x^{i} y^{j}-p_{i j}$, we find the 9 common zeros of the $q_{i j}$, expressed as $\left(x_{k}, x_{k}^{3}\right)(1 \leq i \leq 9)$, with $x_{1} \approx-138.164, x_{2} \approx-1.91764, x_{3} \approx-1.49303, x_{4} \approx-0.711011, x_{5} \approx 0.257975$, $x_{6} \approx 1.16441, x_{7} \approx 1.7846, x_{8} \approx 29.6804, x_{9} \approx 109.398$. Let $\rho_{k}(1 \leq k \leq 9)$ denote the corresponding densities of the representing measure $\mu \equiv \sum_{k=1}^{9} \rho_{k} \delta_{\left(x_{k}, y_{k}\right)}$. To find the densities, we consider the linear system in $\rho_{k}$ defined by $\sum_{k=1}^{9} \rho_{k} x_{k}^{i} y_{k}^{j}=\beta_{i j}$ $(i, j \geq 0, i+j \leq 6)$. This system is very poorly conditioned, but a calculation using Mathematica's LinearSolve yields $\rho_{1} \approx 0$ (order $10^{-23}$ ), $\rho_{1} \approx 0.0203374$, $\rho_{3} \approx 0.131487, \rho_{4} \approx 0.271837, \rho_{5} \approx 0.309585, \rho_{6} \approx 0.205296, \rho_{7} \approx 0.0614581$, $\rho_{8} \approx 0$ (order $10^{-18}$ ), $\rho_{9} \approx 0$ (order $10^{-22}$ ). With these values, $\mu$ interpolates 20 of the 28 moments with high precision, but interpolates very poorly the moments for $y^{4}$ and for monomials of degrees 5 and 6 having degree in $y$ at least 3 . In this case, Theorem 2.4 ensures the existence of a representing measure, but numerical difficulties interfere with a precise calculation of the densities.

\section{A finite variety Case of the $y=x^{3}$ moment problem}

In this section we study the case (1.3) of Section 1, which is used in Section 5 to prove part of Theorem 1.4-iii). We solve the moment problem for $\beta^{(2 n)}$ in the case when $M(n)$ has the column relation

$$
Y=X^{3}
$$


and also a column relation of the form

$$
X Y^{i}=\alpha X^{2} Y^{i-1}+p(X, Y),
$$

where $2 \leq i \leq n-1, \alpha \in \mathbb{R}, p \in \mathcal{P}_{i}$, and the set of columns

$$
\begin{gathered}
\mathcal{S}_{i} \equiv\left\{1, X, Y, X^{2}, X Y, Y^{2}, \ldots, X^{2} Y^{j-2}, X Y^{j-1}, Y^{j},\right. \\
\left.\ldots, X^{2} Y^{i-2}, X Y^{i-1}, Y^{i}, X^{2} Y^{i-1}\right\}
\end{gathered}
$$

is independent. The following result provides part of the proof of Theorem 1.4 (cf. Section 5, Theorem 5.1-iii)).

Theorem 4.1. Suppose $n \geq 3$ and $M(n)$ satisfies (4.1) and (4.2) (for some $i$, $2 \leq i \leq n-1$, with $\mathcal{S}_{i}$ independent). The following are equivalent for $\beta \equiv \beta^{(2 n)}$ :

i) $\beta$ admits a representing measure (necessarily supported in $y=x^{3}$ );

ii) $\beta$ admits a rank $M(n)$-atomic representing measure;

iii) $M(n)$ is positive and recursively generated, and $r \equiv \operatorname{rank} M(n) \leq v \equiv$ $\operatorname{card} \mathcal{V}(M(n))$.

For $n=3$, Theorem 4.1 essentially coincides with [CFM, Theorem. 4.1]. Our proof of Theorem 4.1 generalizes the method of [CFM, Section 4].

We require several preliminary results. The first result does not require (4.2).

Lemma 4.2. Suppose $M(n)$ is recursively generated and $Y=X^{3}$ in $\mathcal{C}_{M(n)}$. If $i, j \geq 0$ and $3+i+j \leq 2 n$, then $\beta_{3+i, j}=\beta_{i, j+1}$.

Proof. Suppose first that $3+i \leq n$, so that $X^{3+i}=X^{i} Y$ by recursiveness. If $j \leq n$, then $\beta_{3+i, j}=\left\langle M(n)\left(x^{3+i}\right)^{\uparrow},\left(y^{j}\right)^{\uparrow}\right\rangle=\left\langle M(n)\left(x^{i} y\right)^{\Upsilon},\left(y^{j}\right)^{\uparrow}\right\rangle=\beta_{i, j+1}$. If $j>n$, then $0<j-n \leq 3+i+j-n \leq n$, so $\beta_{3+i, j}=\left\langle M(n)\left(x^{3+i} y^{j-n}\right)^{2},\left(y^{n}\right)^{r}\right\rangle$ $=\left\langle M(n)\left(x^{i} y^{j-n+1}\right)^{\uparrow},\left(y^{n}\right)^{\uparrow}\right\rangle=\beta_{i, j+1}$. Next, suppose $3+i>n(\geq 3)$. Then $\beta_{3+i, j}=\left\langle M(n)\left(x^{n}\right)^{\Upsilon},\left(x^{3+i-n} y^{j}\right)^{\uparrow}\right\rangle=\left\langle M(n)\left(x^{n-3} y\right)^{\Upsilon},\left(x^{3+i-n} y^{j}\right)^{\uparrow}\right\rangle=\beta_{i, j+1}$.

Suppose $M(n)$ has the column basis

$$
\begin{aligned}
\mathcal{B}=\mathcal{S}_{n-1} \equiv & \left\{1, X, Y, X^{2}, X Y, Y^{2}, \ldots, X^{2} Y^{j-2}, X Y^{j-1}, Y^{j},\right. \\
& \left.\ldots, X^{2} Y^{n-3}, X Y^{n-2}, Y^{n-1}, X^{2} Y^{n-2}\right\} .
\end{aligned}
$$

Thus, in addition to $Y=X^{3}$, there are column relations of the form

$$
X Y^{n-1}=\alpha X^{2} Y^{n-2}+p(X, Y)\left(\alpha \in \mathbb{R}, p \in \mathcal{P}_{n-1}\right)
$$

and

$$
Y^{n}=\gamma X^{2} Y^{n-2}+q(X, Y)\left(\gamma \in \mathbb{R}, q \in \mathcal{P}_{n-1}\right) .
$$

Let $\mathcal{V} \equiv \mathcal{V}(M(n))$, so that

$$
\begin{gathered}
y-x^{3} \mid \mathcal{V} \equiv 0 \\
x y^{n-1}-\alpha x^{2} y^{n-2}-p(x, y) \mid \mathcal{V} \equiv 0
\end{gathered}
$$

and

$$
y^{n}-\gamma x^{2} y^{n-2}-q(x, y) \mid \mathcal{V} \equiv 0
$$


Lemma 4.3. Suppose $M(n)$ satisfies (4.1) and has column basis $\mathcal{B}=\mathcal{S}_{n-1}$. If $\mu$ is a signed measure supported in $\mathcal{V} \equiv \mathcal{V}(M(n))$ which interpolates all monomials corresponding to elements of $\mathcal{B}$, then $\mu$ is an interpolating measure for $\beta^{(2 n)}$; i.e., for $i, j \geq 0$ and $i+j \leq 2 n$,

$$
\int x^{i} y^{j} d \mu=\beta_{i j}
$$

Proof. Since $Y=X^{3},(4.5)$ holds. Since $\mathcal{B}$ is a basis for $\mathcal{C}_{M(n)}$, we have column relations (4.3) and (4.4), so (4.6)-(4.7) also hold. The proof is by induction on the degree $\delta \equiv i+j$ of $x^{i} y^{j}$, and since $\mu$ interpolates the moments corresponding to elements of $\mathcal{B},(4.8)$ holds for $\delta=0,1,2$. Now suppose (4.8) holds for some $\delta$, $2 \leq \delta<n-1$. Since $\mathcal{B}$ contains $X^{2} Y^{k}, X Y^{k+1}, Y^{k+2}$ for $0 \leq k \leq n-3$, to show that (4.8) holds for degree $\delta+1$, it suffices to verify (4.8) for $x^{i} y^{j}$ with $3 \leq i \leq \delta+1$, $j=\delta+1-i$. Now, $\int x^{i} y^{j} d \mu=\int x^{i-3} y^{j+1} d \mu=\beta_{i-3, j+1}$ (from (4.5), and from (4.8) by induction, since $i+j-2<\delta$ ). Lemma 4.2 implies $\beta_{i-3, j+1}=\beta_{i, j}$, so it follows that (4.8) holds for $i+j=\delta+1$. Thus, by induction, (4.8) holds for $i+j \leq n-1$.

Now assume that $n-1 \leq \delta<2 n$ and that (4.8) holds whenever $i+j \leq \delta$. To show that (4.8) holds for $\delta+1$, assume first that $i+j=\delta+1$ with $i \geq 3$. The proof procedes exactly as just above, so we have

$$
\int x^{i} y^{j} d \mu=\beta_{i, j}(i+j=\delta+1 \text { and } i \geq 3) .
$$

Next, consider $x^{2} y^{n+s}$ with $n \leq n+s+2=\delta+1 \leq 2 n$. We have $\int x^{2} y^{n+s} d \mu=$ $\int x y^{s+1}\left(x y^{n-1}\right) d \mu=\int x y^{s+1}\left(\alpha x^{2} y^{n-2}+p(x, y)\right) d \mu$ (from (4.6)) $=\int \alpha x^{3} y^{n+s-1} d \mu$ $+\int x y^{s+1} p(x, y) d \mu=\Lambda_{\beta}\left(\alpha x^{3} y^{n+s-1}\right)+\Lambda_{\beta}\left(x y^{s+1} p\right)$ (by (4.9) for the first term, by (4.8) and induction for the second term, since deg $\left.x y^{s+1} p \leq \delta\right)=$ $\left\langle M(n)\left(\alpha x^{2} y^{n-2}+p\right)^{\uparrow},\left(x y^{s+1}\right)^{\uparrow}\right\rangle=\left\langle M(n)\left(x y^{n-1}\right)^{\uparrow},\left(x y^{s+1}\right)^{\uparrow}\right\rangle($ by $(4.3))=\beta_{2, n+s}$. We now have

$$
\int x^{2} y^{n+s} d \mu=\beta_{2, n+s}(n+1 \leq n+s+2=\delta+1 \leq 2 n) .
$$

We next consider $x y^{n+t}$, where $n \leq n+t+1=\delta+1 \leq 2 n$. We have $\int x y^{n+t} d \mu=\int y^{t+1}\left(x y^{n-1}\right) d \mu=\int y^{t+1}\left(\alpha x^{2} y^{n-2}+p(x, y)\right) d \mu \quad$ (from (4.6)) $=$ $\int \alpha x^{2} y^{n+t-1} d \mu+\int y^{t+1} p d \mu=\Lambda_{\beta}\left(\alpha x^{2} y^{n+t-1}\right)+\Lambda_{\beta}\left(y^{t+1} p\right)$ (by (4.10) for the first term (with $s=t-1$ ), by (4.8) and induction for the second term, since deg $y^{t+1} p \leq$ $\delta)=\left\langle M(n)\left(\alpha x^{2} y^{n-2}+p\right)^{-},\left(y^{t+1}\right)^{\uparrow}\right\rangle=\left\langle M(n)\left(x y^{n-1}\right)^{\Upsilon},\left(y^{t+1}\right)^{\uparrow}\right\rangle($ by $(4.3))=\beta_{1, n+t}$. Thus,

$$
\int x y^{n+t} d \mu=\beta_{1, n+t}(n \leq n+t+1=\delta+1 \leq 2 n) .
$$

Finally, we consider $y^{n+r}$ with $n \leq n+r=\delta+1 \leq 2 n$. We have $\int y^{n+r} d \mu$ $=\int\left(\gamma x^{2} y^{n-2}+q(x, y)\right) y^{r} d \mu$ (from $\left.(4.7)\right)=\Lambda_{\beta}\left(\gamma x^{2} y^{n+r-2}\right)+\Lambda_{\beta}\left(y^{r} q\right)$ (by (4.10) with $s=r-2$ for the first term, by induction with (4.8) for the second term, since $\left.\left.\operatorname{deg} y^{r} q \leq \delta\right)=\left\langle M(n)\left(\gamma x^{2} y^{n-2}+q\right)\right)^{r},\left(y^{r}\right)^{\Upsilon}\right\rangle=\left\langle M(n) \widehat{y^{n}}, \widehat{y^{r}}\right\rangle$ (by $\left.(4.4)\right)=\beta_{0, n+r}$. Thus,

$$
\int y^{n+r} d \mu=\beta_{0, n+r}(n \leq n+r=\delta+1 \leq 2 n) .
$$

In view of (4.9)-(4.12), we conclude that (4.8) holds for $\delta+1$; the proof is complete. 
Proof of Theorem 4.1. Since ii) and (1.5) imply i), and i) always implies iii) (cf. Section 1), it suffices to show that iii) implies ii). Let $f(x, y)=x y^{i}-\alpha x^{2} y^{i-1}-$ $p(x, y)$ and let $g(x)=f\left(x, x^{3}\right)$. From (4.1) and (4.2), we have $v \leq \operatorname{deg} g=3 i+1$. Since $\mathcal{S}_{i}$ is independent and $M(n) \succeq 0$, the Extension Principle implies that $3 i+1=$ card $\mathcal{S}_{i} \leq \operatorname{rank} M(i+1) \leq r \leq v \leq 3 i+1$, whence $M(n)$ is extremal, with

$$
\operatorname{rank} M(n)=\operatorname{card} \mathcal{V}(M(n))=3 i+1=\operatorname{rank} M(i+1)=\operatorname{card} \mathcal{S}_{i} .
$$

If $i+1<n$, then since $\operatorname{rank} M(i+1)=\operatorname{rank} M(n), M(n)$ has flat data, so Theorem 1.6 implies that $\beta$ has a (unique, $\operatorname{rank} M(n)$-atomic) representing measure.

Assume now that $i+1=n$, so (4.13) implies that $r=v=3 n-2$, whence $\mathcal{B} \equiv \mathcal{S}_{n-1}$ is a basis for $\mathcal{C}_{M(n)}$. We will show that $\beta$ is consistent, and thereby derive the existence of a representing measure from [CFM, Theorem 2.8]. Let $\mathcal{V} \equiv\left\{\left(x_{i}, x_{i}^{3}\right)\right\}_{i=1}^{3 n-2}$ denote the distinct points of $\mathcal{V} \equiv \mathcal{V}(M(n))$, and note that the $x_{i}$ 's are necessarily distinct. We claim that $\mathcal{K}_{n}:=\left\{p \in \mathcal{P}_{n}: p \mid \mathcal{V} \equiv 0\right\}$ satisfies $\operatorname{dim} \mathcal{K}_{n} \leq \operatorname{dim} \mathcal{P}_{n}-v$. For each $i, 3 \leq i \leq n$, consider the $i-2$ monomials $x^{i}, x^{i-1} y, \ldots, x^{3} y^{i-3}$. Under the substitution, $y=x^{3}$, these are precisely the monomials in $\mathcal{P}_{n}$ which, when restricted to $\mathcal{V}$, agree with monomials of strictly lower degree; indeed, for $3 \leq p \leq i \leq n, x^{p} y^{i-p}\left|\mathcal{V} \equiv x^{p-3} y^{i-p+1}\right| \mathcal{V}$. Let $\mathcal{T}$ denote the collection of all such monomials in $\mathcal{P}_{n}$, together with $x y^{n-1}$ and $y^{n}$. The latter two monomials are the only monomials in $\mathcal{P}_{n}$ which, under the substitution $y=x^{3}$, have degree in $x$ exceeding $v-1$. Note that

$$
\tau \equiv \operatorname{card} \mathcal{T}=\frac{1}{2}\left(n^{2}-3 n+6\right)=\operatorname{dim} \mathcal{P}_{n}-v
$$

Consider the mapping $\Psi: \mathcal{K}_{n} \longrightarrow \mathbb{R}^{\tau}$ defined as follows. For

$$
p(x, y) \equiv \sum_{i, j \geq 0, i+j \leq n} c_{i j} x^{i} y^{j} \in \mathcal{K}_{n},
$$

let

$$
\Psi(p)=\left(c_{3,0}, c_{4,0}, c_{3,1}, \ldots, c_{i, 0}, \ldots, c_{3, i-3}, \ldots, c_{n, 0}, \ldots, c_{3, n-3}, c_{1, n-1}, c_{0, n}\right),
$$

the vector of coefficients corresponding to monomials in $\mathcal{T}$. We assert that $\Psi$ is $1-1$. For suppose $\Psi(p)=0$, i.e., $c_{i j}=0$ if $x^{i} y^{j} \in \mathcal{T}$. Then $p(x, y)=$ $\sum_{x^{i} y^{j} \in \mathcal{P}_{n} \backslash \mathcal{T}} c_{i j} x^{i} y^{j}=c_{2, n-2} x^{2} y^{n-2}+c_{0, n-1} y^{n-1}+c_{1, n-2} x y^{n-2}+c_{2, n-3} x^{2} y^{n-3}+$ $\cdots+$ (terms of lower degree). Thus, $q(x):=p\left(x, x^{3}\right)$ satisfies $\operatorname{deg} q \leq 3 n-3$. Since $p \mid \mathcal{V} \equiv 0$, we have $q\left(x_{i}\right)=0(1 \leq i \leq 3 n-2)$, whence $q \equiv 0$ and $c_{i j}=0$ for all $x^{i} y^{j} \in \mathcal{P}_{n} \backslash \mathcal{T}$. Thus, $p \equiv 0$, so $\Psi$ is $1-1$ and $\operatorname{dim} \mathcal{K}_{n} \leq \operatorname{dim} \mathcal{P}_{n}-v$.

Since $\operatorname{dim} \mathcal{K}_{n} \leq \operatorname{dim} \mathcal{P}_{n}-v$, it follows from [CFM, Lemma 4.3] that $\beta$ is extremal and weakly consistent, i.e., $p \in \mathcal{P}_{n}, p \mid \mathcal{V} \equiv 0 \Longrightarrow p(X, Y)=0$ in $\mathcal{C}_{M(n)}$. Consider the basis $\mathcal{B}$ for $\mathcal{C}_{M(n)}$. Since $\beta$ is extremal and weakly consistent, CFM, Lemma 2.7 and (2.5)] implies that there is a signed measure $\mu_{\mathcal{B}}$ such that supp $\mu_{\mathcal{B}}=\mathcal{V}$ and $\int x^{i} y^{j} d \mu_{\mathcal{B}}=\beta_{i j}$ whenever $X^{i} Y^{j} \in \mathcal{B}$. Lemma 4.3 now implies that $\mu_{\mathcal{B}}$ is an interpolating measure for $\beta$, whence [CFM, Theorem 2.8] implies that $\mu_{\mathcal{B}}$ is actually a representing measure for $\beta$. The proof is now complete.

\section{Solution of the $y=x^{3}$ truncated moment PRoblem}

In this section we provide concrete necessary and sufficient conditions for $\Gamma$ representing measures. Theorem 5.1 (which restates Theorem 1.4) treats the case $n \geq 3$. In Propositions 5.5-5.9 we treat the cases $n=1$ and $n=2$. In the sequel, for $1 \leq i \leq n, \mathcal{P}_{i}[X, Y]$ denotes the linear span in $\mathcal{C}_{M(n)}$ of the set $\left\{p(X, Y): p \in \mathcal{P}_{i}\right\}$. 
Suppose $M(n)$ is recursively generated and $Y=X^{3}$ in $\mathcal{C}_{M(n)}$. It follows that for $1 \leq i<n, X^{k} Y^{i+1-k} \in \mathcal{P}_{i}[X, Y](3 \leq k \leq i+1)$. If there is a dependence relation in $\mathcal{P}_{i+1}[X, Y]$ involving $X^{2} Y^{i-1}, X Y^{i}$, or $Y^{i+1}$, then exactly one of the following cases holds:

$$
\begin{gathered}
X^{2} Y^{i-1} \in \mathcal{P}_{i}[X, Y], \\
X^{2} Y^{i-1} \notin \mathcal{P}_{i}[X, Y] \text { and } X Y^{i} \in \operatorname{span}\left\{X^{2} Y^{i-1}, \mathcal{P}_{i}[X, Y]\right\} \\
X^{2} Y^{i-1} \notin \mathcal{P}_{i}[X, Y], X Y^{i} \notin \operatorname{span}\left\{X^{2} Y^{i-1}, \mathcal{P}_{i}[X, Y]\right\} \\
\text { and } Y^{i+1} \in \operatorname{span}\left\{X^{2} Y^{i-1}, X Y^{i}, \mathcal{P}_{i}[X, Y]\right\}
\end{gathered}
$$

Theorem 5.1. Let $n \geq 3$. If $\beta \equiv \beta^{(2 n)}$ has a representing measure supported in $y=$ $x^{3}$, then $M(n)$ is positive and recursively generated, rank $M(n) \leq \operatorname{card} \mathcal{V}(M(n))$, and

$$
Y=X^{3} \text { in } \mathcal{C}_{M(n)}
$$

Conversely, suppose $M(n)$ satisfies all of the preceding conditions, so that any representing measure is necessarily supported in $y=x^{3}$.

i) If $M(2)$ is singular, then $\beta$ has a representing measure.

ii) If $M(n)$ is $\left(y-x^{3}\right)$-pure, then $\beta$ has a representing measure if and only if $\beta_{1,2 n-1}>\psi(\beta)$.

If neither (i) nor (ii) holds, there is a minimal $i, 2 \leq i<n$, such that exactly one of (5.1)-(5.3) holds.

iii) If (5.1) or (5.2) holds, then $\beta$ has a representing measure.

iv) Suppose neither (5.1) nor (5.2) holds, but (5.3) holds for some minimal $i$. If $i \leq n-3$, then $\beta$ admits a representing measure. If $i=n-2$, then $M(n)$ admits a recursively determined extension $M(n+1)$ and $\beta$ has a representing measure if and only if rank $M(n)=\operatorname{rank} M(n-1)$ or rank $M(n+1)=\operatorname{rank} M(n)$. If $i=n-1$, then $M(n)$ is recursively determinate and $\beta$ admits a representing measure if and only if $M(n)$ admits positive, recursively determined extensions $M(n+1), M(n+2)$ satisfying rank $M(n+1)=\operatorname{rank} M(n)$ or rank $M(n+2)=\operatorname{rank} M(n+1)$.

Proof. The necessity of the conditions is clear, and it follows from (1.5) that if $\beta$ has a representing measure supported in $y=x^{3}$, then $Y=X^{3}$ in $\mathcal{C}_{M(n)}$. For the converse, suppose that $M(n)$ is positive, recursively generated, rank $M(n) \leq$ card $\mathcal{V}(M(n))$, and $Y=X^{3}$ in $\mathcal{C}_{M(n)}$. For i), suppose $M(2)$ is singular, with a column relation $q(X, Y)=0$ for some $q \in \mathcal{P}_{2}$. Since $M(n) \succeq 0$, the Extension Principle implies $q(X, Y)=0$ in $\mathcal{C}_{M(n)}$. The existence of a representing measure now follows from Theorem 1.2. For ii), the result follows directly from Theorem 1.1. If neither i) nor ii) holds, there is a minimal $i, 2 \leq i \leq n-1$, such that exactly one of (5.1), (5.2), or (5.3) holds. For case iii), suppose first that (5.1) holds, i.e., the first dependence relation that cannot be derived from (5.4) via recursiveness is of the form $X^{2} Y^{i-1}=p(X, Y)$ for some $p \in \mathcal{P}_{i}$. Let $q(x, y):=$ $x^{2} y^{i-1}-p(x, y)$ and set $g(x):=q\left(x, x^{3}\right)$. We have $3 i=\operatorname{rank} M(i) \leq \operatorname{rank} M(n) \leq$ card $\mathcal{V}(M(n)) \leq \operatorname{deg} g \leq 3 i$, whence $M(n)$ is a flat extension of $M(i)$, so the existence of a representing measure follows from Theorem 1.6. To complete case iii), if (5.2) holds, the existence of a representing measure follows from Theorem 4.1 .

To prove iv), we note that in the remaining case, $X^{2} Y^{i-1} \notin \mathcal{P}_{i}[X, Y], X Y^{i} \notin$ $\operatorname{span}\left\{X^{2} Y^{i-1}, \mathcal{P}_{i}[X, Y]\right\}$, and $Y^{i+1} \in \operatorname{span}\left\{X^{2} Y^{i-1}, X Y^{i}, \mathcal{P}_{i}[X, Y]\right\}$. It follows 
that $\operatorname{rank} M(i+1)=\operatorname{rank} M(i)+2=3 i+2$. Further, there is a column relation in $M(i+1)$ of the form $Y^{i+1}=\alpha X^{2} Y^{i-1}+\gamma X Y^{i}+q(X, Y)$, with $q \in \mathcal{P}_{i}$. Let $g(x, y):=y^{i+1}-\left(\alpha x^{2} y^{i-1}+\gamma x y^{i}+q(x, y)\right)$, so that $\operatorname{card} \mathcal{V}(M(i+1)) \leq$ $\operatorname{deg} g\left(x, x^{3}\right)=3 i+3$. Suppose first that $i+1 \leq n-2$. Since $M(n)$ is positive, the Extension Principle implies that $3 i+2 \leq \operatorname{rank} M(i+1) \leq \operatorname{rank} M(n-2) \leq$ rank $M(n-1) \leq \operatorname{rank} M(n) \leq \operatorname{card} \mathcal{V}(M(n)) \leq \operatorname{card} \mathcal{V}(M(i+1)) \leq 3 i+3$. So either $\operatorname{rank} M(n-2)=\operatorname{rank} M(n-1)$ or rank $M(n-1)=\operatorname{rank} M(n)$, and the existence of a representing measure follows from the case of flat data (cf. Theorem 1.6). Suppose next that $i+1=n-1$. Relation (5.3) and [F4, Prop. 4.2] imply that $M(n-1)$ is recursively determinate, and $M(n)$ is a positive, recursively generated extension of $M(n-1)$. Since $\operatorname{card} \mathcal{V}(M(n-1))-\operatorname{rank} M(n-1) \leq$ $(3 n-3)-(3 n-4)=1$, it follows from Theorem 1.7 (applied to $M(n-1))$ that $\beta$ admits a representing measure if and only if $\operatorname{rank} M(n)=\operatorname{rank} M(n-1)$, or the recursively determined extension $M(n+1)$ satisfies $\operatorname{rank} M(n+1)=\operatorname{rank} M(n)$. Finally, suppose $i+1=n$. Again, [F4, Prop.4.2] implies that $M(n)$ is recursively determinate and $\operatorname{card} \mathcal{V}(M(n))-\operatorname{rank} M(n) \leq 3 n-(3 n-1)=1$, so Theorem 1.7 implies that $\beta$ has a representing measure if and only if $M(n)$ admits positive, recursively determined extensions $M(n+1)$ and $M(n+2)$ satisfying $\operatorname{rank} M(n+1)=$ $\operatorname{rank} M(n)$ or $\operatorname{rank} M(n+2)=\operatorname{rank} M(n+1)$.

Several examples of [CF7] illustrate case i) of Theorem 5.1. Example 3.2 (above) illustrates case ii). The flat extension $M(4)$ in Example 3.2 illustrates (5.2) in case iii), while [CFM, Example 4.2] illustrates (5.3) in case iii). In case iv) of Theorem 5.1 , the existence or nonexistence of the extensions $M(n+1)$ and $M(n+2)$ (and the existence of a representing measure) can be determined from the algorithm presented in [F4, Algorithm 4.10], which shows how to implement Theorem 1.7. [F4, Example 4.18] illustrates iv) in a case where $n=3, i=2, r=v=8$, but the recursively determined extension $M(4)$ is not positive, so there is no representing measure. [F4, Example 3.3] illustrates iv) in a case with $n=3, i=2, r=8$, and $v=9$, so in principle the recursively determined extensions $M(4)$ and $M(5)$ are required. However, in this example, $M(4)$ is already a flat extension of $M(3)$, so the existence of a representing measure is established without recourse to $M(5)$. We next illustrate Theorem 5.1-iv) in a case where $n=3, i=2, r=8, v=9$, and both $M(4)$ and $M(5)$ are used to determine whether or not $\beta^{(6)}$ has a representing measure.

Example 5.2. Let $n=3$ and consider

$$
M(3)=\left(\begin{array}{cccccccccc}
1 & 0 & 0 & 1 & 2 & 5 & 0 & 0 & 0 & 0 \\
0 & 1 & 2 & 0 & 0 & 0 & 2 & 5 & 14 & 42 \\
0 & 2 & 5 & 0 & 0 & 0 & 5 & 14 & 42 & 132 \\
1 & 0 & 0 & 2 & 5 & 14 & 0 & 0 & 0 & 0 \\
2 & 0 & 0 & 5 & 14 & 42 & 0 & 0 & 0 & 0 \\
5 & 0 & 0 & 14 & 42 & 132 & 0 & 0 & 0 & 0 \\
0 & 2 & 5 & 0 & 0 & 0 & 5 & 14 & 42 & 132 \\
0 & 5 & 14 & 0 & 0 & 0 & 14 & 42 & 132 & 429 \\
0 & 14 & 42 & 0 & 0 & 0 & 42 & 132 & 429 & 2000 \\
0 & 42 & 132 & 0 & 0 & 0 & 132 & 429 & 2000 & 338881
\end{array}\right) .
$$


We have $M(3) \succeq 0, M(2) \succ 0$, and $\operatorname{rank} M(3)=8$, with column relations

$$
Y=X^{3}
$$

and

$$
Y^{3}=q(X, Y),
$$

where $q(x, y):=-2285 x+5720 y-34441 x^{2} y+578 x y^{2}$. Let $r_{1}(x, y)=y-x^{3}$ and $r_{2}(x, y)=y^{3}+2285 x-5720 y+3441 x^{2} y-578 x y^{2}$. Then $\mathcal{V} \equiv \mathcal{V}(M(3))=\mathcal{Z}_{r_{1}} \cap \mathcal{Z}_{r_{2}}$, and a calculation shows that $\mathcal{V}=\left\{\left(x_{i}, x_{i}^{3}\right)\right\}_{i=1}^{9}$, where $x_{1}=0, x_{2} \approx 0.765081$, $x_{3} \approx 1.4139, x_{4} \approx 1.84764, x_{5} \approx 23.9166$, and $x_{5+i}=-x_{i+1}(1 \leq i \leq 4) . M(3)$ satisfies the hypothesis of Theorem 5.1-iv) with $i=n-1$, so we proceed to generate the recursively determined extension $M(4)$. This extension is uniquely determined by imposing the column relations $X^{4}=X Y, X^{3} Y=Y^{2}, X Y^{3}=(x q)(X, Y)$,

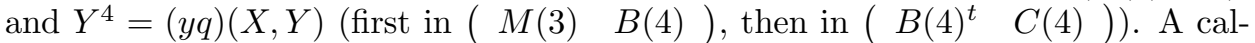
culation shows that these relations unambiguously define a positive moment matrix $M(4)$ with rank $M(4)=9$. It follows that $M(3)$ admits no flat extension, so we attempt to construct the extension $M(5)$, uniquely determined by imposing the relations $X^{5}=X^{2} Y, X^{4} Y=X Y^{2}, X^{3} Y^{2}=Y^{3}, X^{2} Y^{3}=\left(x^{2} q\right)(X, Y)$, $X Y^{4}=(x y q)(X, Y), Y^{5}=\left(y^{2} q\right)(X, Y)$. A calculation of these columns (first in $(M(4) B(5))$, then in $\left.\left(B(5)^{t} C(5)\right)\right)$, shows that they do fit together to unambiguously define a moment matrix $M(5)$. From the form of $q(x, y)$, we see that $M(5)$ is actually a flat extension of $M(4)$. For example, in $\mathcal{C}_{M(5)}$ we have $X^{2} Y^{3}=\left(x^{2} q\right)(X, Y)=-2285 X^{3}+5720 X^{2} Y-34441 X^{4} Y+578 X^{3} Y^{2}=$ $-2285 Y+5720 X^{2} Y-34441 X Y^{2}+578 q(X, Y)$, and each column in the last expression has degree at most 3. Corresponding to this flat extension is the unique representing measure $\mu \equiv \mu_{M(5)}$ described in Section 1 (cf. Theorem 1.6). Clearly, supp $\mu=\mathcal{V}$, so $\mu$ is of the form $\mu=\sum_{i=1}^{9} \rho_{i} \delta_{\left(x_{i}, x_{i}^{3}\right)}$. To compute the densities, we use (1.7) with the column basis $\mathcal{B} \equiv\left\{1, X, Y, X^{2}, X Y, Y^{2}, X^{2} Y, X Y^{2}, X^{2} Y^{2}\right\}$ for $M(4)$, and we find $\rho_{1} \approx 0.249891, \rho_{2}=\rho_{6} \approx 0.21335, \rho_{3}=\rho_{7} \approx 0.125055$, $\rho_{4}=\rho_{8} \approx 0.0366505$, and $\rho_{5}=\rho_{9} \approx 0$ (order $\left.10^{-20}\right)$. Thus, the existence of a representing measure for $\beta^{(6)}$ is established on the basis of the extensions $M(4)$ and $M(5)$, in keeping with Theorem 5.1-iv). (Since $M(4)$ is extremal, instead of computing the flat extension $M(5)$, we could have established the existence of a representing measure by showing that $\beta^{(6)}$ is consistent, but this entails a matrix calculation based on an application of [F3, Prop. 3.6].)

As an application of Theorem 5.1, we have the following criterion for $\Gamma$-representing measures, expressed in terms of moment matrix extensions.

Corollary 5.3. Let $n \geq 3$ and suppose $Y=X^{3}$ in $\mathcal{C}_{M(n)} . \beta \equiv \beta^{(2 n)}$ has a $\Gamma$ representing measure if and only if $M(n)$ admits a positive, recursively generated extension $M(n+2)$.

Proof. The necessity of the condition is clear from (1.5) and $[\mathrm{BT}$. For sufficiency, we consider the types of dependence relations that may be present in $\mathcal{C}_{M(n)}$. If there is a nonzero $q \in \mathcal{P}_{2}$ such that $q(X, Y)=0$ (equivalently, if $M(2)$ is singular), then the existence of a positive, recursively generated extension $M(n+1)$ is sufficient for a $\Gamma$-representing measure (cf. Theorem 1.2). Similarly, in the case when $M(n)$ is $\left(y-x^{3}\right)$-pure, Theorem 1.1-iii) shows that if there is a positive, recursively generated extension $M(n+1)$, then $\beta$ has a $\Gamma$-representing measure. 
In the remaining case, recursiveness implies that at least one of the following relations holds in $\mathcal{C}_{M(n)}$ :

$$
\begin{gathered}
Y^{n}=\alpha X Y^{n-1}+\delta X^{2} Y^{n-2}+q(X, Y)\left(q \in \mathcal{P}_{n-1}\right), \\
X Y^{n-1}=\delta X^{2} Y^{n-2}+q(X, Y)\left(q \in \mathcal{P}_{n-1}\right), \\
X^{2} Y^{n-2}=q(X, Y)\left(q \in \mathcal{P}_{n-1}\right) .
\end{gathered}
$$

Consider the case when (5.5) holds. Since $Y=X^{3}$, recursiveness implies that in $\mathcal{C}_{M(n+2)}$ we have $X^{n+2}=X^{n-1} Y, \ldots, X^{3} Y^{n-1}=Y^{n}$. Further, $X^{2} Y^{n}=$ $\alpha Y^{n}+\delta X Y^{n-1}+\left(x^{2} q\right)(X, Y) \in \mathcal{P}_{n+1}[X, Y]$, whence $X Y^{n+1}=\alpha X^{2} Y^{n}+\delta Y^{n}+$ $(x y q)(X, Y) \in \mathcal{P}_{n+1}[X, Y]$, and thus $Y^{n+2}=\alpha X Y^{n+1}+\delta X^{2} Y^{n}+\left(y^{2} q\right)(X, Y) \in$ $\mathcal{P}_{n+1}[X, Y]$. The preceding relations show that $M(n+2)$ is a flat extension of $M(n+1)$, so the existence of a representing measure (necessarily supported in $\Gamma$ ) follows from Theorem 1.6.

Next suppose that (5.6) holds. In $M(n+1)$, we then have

$$
X^{2} Y^{n-1}=\delta Y^{n-1}+(x q)(X, Y) \in \mathcal{P}_{n}[X, Y] .
$$

We may express $q(X, Y)$ as $q(X, Y)=a Y^{n-1}+b X Y^{n-2}+c X^{2} Y^{n-3}+r(X, Y)(r \in$ $\left.\mathcal{P}_{n-2}\right)$, so in $M(n+2)$, we have

$$
Y^{n}=X^{3} Y^{n-1}=\delta X Y^{n-1}+a X^{2} Y^{n-1}+b Y^{n-1}+c X Y^{n-2}+\left(x^{2} r\right)(X, Y) .
$$

Now, (5.8) and (5.9) imply that relation (5.5) holds in $M(n)$, so the existence of a representing measure follows from the previous case.

To complete the proof, we consider the case when relation (5.7) holds, and we may express this relation as

$$
X^{2} Y^{n-2}=\alpha Y^{n-1}+\delta X Y^{n-2}+\gamma X^{2} Y^{n-3}+r(X, Y)\left(r \in \mathcal{P}_{n-2}\right) .
$$

In $M(n+1)$, we have

$$
Y^{n-1}=\alpha X Y^{n-1}+\delta X^{2} Y^{n-2}+\gamma Y^{n-2}+(x r)(X, Y) .
$$

If $\alpha \neq 0,(5.11)$ may be reduced to the form of (5.6), so we may assume $\alpha=0$. Writing $r(X, Y)=a Y^{n-2}+b X Y^{n-3}+c X^{2} Y^{n-4}+s(X, Y)\left(s \in \mathcal{P}_{n-3}, c=0\right.$ if $n=$ 3), (5.11) (with $\alpha=0$ ) implies $X Y^{n-1}=\delta Y^{n-1}+\gamma X Y^{n-2}+a X^{2} Y^{n-2}+b Y^{n-2}+$ $c X Y^{n-3}+\left(x^{2} s\right)(X, Y)$, so we may again reduce to the case when (5.6) holds.

The preceding result yields a degree-bounded weighted sum-of-squares representation for polynomials that are strictly positive on $\Gamma$.

Corollary 5.4. Let $n \geq 3$. If $r(x, y) \in \mathcal{P}_{2 n}$ is strictly positive on $\Gamma$, then $r$ admits a decomposition of the form $r(x, y)=\sum f_{i}(x, y)^{2}+\left(y-x^{3}\right) \sum g_{i}(x, y)^{2}$, where $\operatorname{deg} f_{i}^{2}, \operatorname{deg}\left(y-x^{3}\right) g_{i}^{2} \leq 2(n+3)$.

Proof. Corollary 5.3 and $[\mathrm{CF} 4$, Proposition 3.9] together imply that $\Gamma$ satisfies property $\left(R_{n, 2}\right)$ of [CF8, so the result follows from [CF8, Theorem 1.5].

In Section 6 we provide analogues of Corollaries 5.3 and 5.4 for the curves $y=$ $g(x)$ and $y g(x)=1$. However, in Proposition 6.1 (the analogue of Corollary 5.3), we generally require extensions $M(n+k)$ where $k$ depends on $d e g g$.

We conclude this section by considering the truncated $\Gamma$-moment problem for $\beta^{(2 n)}$ when $n=1$ or $n=2$. We normalize $\beta_{0,0}=1$. For $M(2)$ to have a representing measure supported in $y=x^{3}$ (and hence a positive extension $M(3)$ with $Y=X^{3}$ ), 
we must have $b \equiv \beta_{0,1}=\beta_{3,0}, d \equiv \beta_{0,2}=\beta_{3,1}, e=\beta_{1,1}=\beta_{4,0}$. Thus, $M(2)$ is of the form

$$
M(2)=\left(\begin{array}{llllll}
1 & a & b & c & e & d \\
a & c & e & b & g & h \\
b & e & d & g & h & j \\
c & b & g & e & d & f \\
e & g & h & d & f & p \\
d & h & j & f & p & q
\end{array}\right) .
$$

In the sequel, $M_{4}$ denotes the $4 \times 4$ upper left-hand block in $M(2)$. A positive, recursively generated extension $M(3)$ with $X^{3}=1$ must have block $B(3)$ of the form

$$
B(3)=\left(\begin{array}{llll}
b & g & h & j \\
e & d & f & p \\
d & f & p & q \\
g & h & j & A \\
h & j & A & B \\
j & A & B & C
\end{array}\right) .
$$

For the case when $M(2) \succ 0$ (positive definite), we adapt the notation of Section 2 and the proof of Theorem 2.4. We set $J=M(2)$ and write $J^{-1} \equiv\left(\begin{array}{cc}P & v \\ v^{t} & \epsilon\end{array}\right)$ and $X^{2} Y \equiv\left(\begin{array}{c}w \\ A\end{array}\right)$ in $\mathcal{C}_{B(3)}$. In the flat extension $M^{\mathrm{b}} \equiv[M(2) ; B(3)]$ we have $C^{b} \equiv B(3)^{t} J^{-1} B(3)=\left(c_{i, j}\right)_{1 \leq i, j \leq 4}$, and the conditions for a flat moment matrix extension $M(3)$ are $c_{2,2}=c_{3,1}\left(=\beta_{4,2}=\beta_{1,3}=p\right), c_{3,2}=c_{4,1}\left(=\beta_{3,3}=\beta_{0,4}=q\right)$, and $c_{4,2}=c_{3,3}$. As in the proof of Theorem 2.4, we set $\psi(\beta)=\frac{\omega \epsilon-\langle v, w\rangle^{2}}{\epsilon}$, where $\omega=\langle P w, w\rangle$. A straightforward adaptation of the proof of Theorem 2.4 yields the following result.

Proposition 5.5. If $M(2) \succ 0$, then $\beta \equiv \beta^{(4)}$ has a representing measure supported in $y=x^{3}$ if and only if $p \equiv \beta_{1,3}>\psi(\beta)$, in which case $\beta$ admits a 6 -atomic $\Gamma$ representing measure (corresponding to a flat extension $M(3)$ ).

We next consider the case when $n=1$ and $M(1) \succ 0$, so that $c>a^{2}$. Let $\gamma:=\frac{c^{3}-2 a b c+b^{2}}{c-a^{2}}$.

Proposition 5.6. Let $\beta \equiv \beta^{(2)}$ and suppose $M(1) \succ 0$.

i) If $e \equiv \beta_{1,1}<\gamma$, then $\beta$ admits no $\Gamma$-representing measure.

ii) If $e>\gamma$, then $\beta$ admits a 6 -atomic $\Gamma$-representing measure.

iii) If $e=\gamma$, there is a unique flat extension of $M(1)$ to $M_{4}$, defined by a column relation $X^{2}=c_{1} 1+c_{2} X+c_{3} Y . \beta$ has a $\Gamma$-representing measure if and only if $\sigma:=\left\{\left(x, x^{3}\right): x^{2}=c_{1}+c_{2} x+c_{3} x^{3}\right\}$ has exactly 3 points, $\left\{\left(x_{i}, y_{i}\right)\right\}_{1 \leq i \leq 3}$, the matrix $V$ in (5.17) is invertible, and the measure $\mu \equiv \sum_{i=1}^{3} \rho_{i} \delta_{\left(x_{i}, y_{i}\right)}$ (defined in (5.18)) interpolates the moments $c, d$, and e.

Proof. If $\beta$ admits a $\Gamma$-representing measure, then $\beta$ admits a finitely atomic $\Gamma$ representing measure $\nu$ and $M(2)[\nu]$ must be of the form (5.12). In the sequel we use Smuljan's criterion for positive extensions of matrices (cf. Section 1). A 
calculation shows that $M(1)$ admits an extension to a positive matrix

$$
M_{4} \equiv\left(\begin{array}{cccc}
1 & a & b & c \\
a & c & e & b \\
b & e & d & g \\
c & b & g & e
\end{array}\right)
$$

if and only if $e \geq \gamma$. Thus, if $e<\gamma$, then $M(1)$ admits no $\Gamma$-representing measure.

Next, suppose $e>\gamma$. In this case, there exists $g$ such that $M_{4} \succ 0$. By choosing $h$ arbitrarily and then $f$ sufficiently large, we can ensure that $M_{5}$, the $5 \times 5$ upper left-hand block in (5.12), is positive definite. A calculation shows that for $M(2)$ as in $(5.12), \psi\left(\beta^{(4)}\right)$ is independent of $p$ and $q$. Thus, if we choose $j$ arbitrarily, then choose $p>\psi\left(\beta^{(4)}\right)$, and finally choose $q$ sufficiently large, we can ensure that $M(2)$ satisfies the hypothesis and sufficiency condition of Proposition 5.5. It follows that $\beta^{(4)}$ (and hence $\beta^{(2)}$ ) admits a 6 -atomic $\Gamma$-representing measure.

We now consider the case $e=\gamma$. A calculation shows that in this case there is a unique $g$ such that $M_{4}$ is positive and that with this choice, $\operatorname{rank} M_{4}=$ rank $M(1)=3$. In $M_{4}$ we thus have a column relation of the form

$$
X^{2}=c_{1} 1+c_{2} X+c_{3} Y
$$

If $\mu$ is a finitely atomic $\Gamma$-representing measure, then, by the uniqueness of $g, M_{4}$ is a positive block in $M(2)[\mu]$, so in $M(2)[\mu]$ we have $\beta_{2,1}=g$ and relation (5.15) holds. Thus, by the Extension Principle, (5.15) holds in the column space of $M(3)[\mu]$, as does $Y=X^{3}$, whence supp $\mu$ is contained in

$$
\sigma \equiv\left\{\left(x, x^{3}\right): x^{2}=c_{1}+c_{2} x+c_{3} x^{3}\right\} .
$$

Thus, $3=\operatorname{rank} M(1) \leq \operatorname{rank} M(2)[\mu] \leq \operatorname{rank} M(3)[\mu] \leq \operatorname{card}$ supp $\mu \leq 3$, so supp $\mu=\sigma$ and $\sigma$ consists of exactly 3 distinct points. Let $\sigma=\left\{\left(x_{i}, y_{i}\right)\right\}_{1 \leq i \leq 3}$, so that $\mu \equiv \sum_{i=1}^{3} \rho_{i} \delta_{\left(x_{i}, y_{i}\right)}$. Consider

$$
V=\left(\begin{array}{ccc}
1 & 1 & 1 \\
x_{1} & x_{2} & x_{3} \\
y_{1} & y_{2} & y_{3}
\end{array}\right)
$$

If $V^{t}$ is singular, then there is a polynomial $d_{1}+d_{2} x+d_{3} y$ which vanishes on supp $\mu$, whence $d_{1}+d_{2} X+d_{3} Y=0$ in $\mathcal{C}_{M(1)}$, contradicting $M(1) \succ 0$. Thus $V$ is invertible and $\rho \equiv\left(\rho_{1}, \rho_{2}, \rho_{3}\right)$ is uniquely determined by

$$
\rho=V^{-1}(1, a, b) \text {. }
$$

We conclude that $\beta$ has a $\Gamma$-representing measure if and only if card $\sigma=3, V$ is invertible, and the measure defined by (5.18) is a representing measure (i.e., the measure interpolates moments $b, c$, and $d$ ).

We next consider the case when $M(2)$ is positive and singular, with $M(1)$ positive definite.

Proposition 5.7. Let $\beta \equiv \beta^{(6)}$ and suppose that $M(2)$ is positive and singular, with $M(1) \succ 0$.

i) If rank $M(2)=3$, so that $M(2)$ is a flat extension of $M(1)$, then $\beta$ has a $\Gamma$-representing measure if and only if the unique, 3-atomic, representing measure associated with $M(2)$ is supported in $\Gamma$.

ii) If rank $M(2) \geq 4$ and there is a column relation $X^{2}=d_{1} 1+d_{2} X+d_{3} Y$, then there is no $\Gamma$-representing measure. 
iii) If rank $M(2)=4$ and there are column relations $X Y=r_{1} 1+r_{2} X+r_{3} Y+$ $r_{4} X^{2}$ and $Y^{2}=s_{1} 1+s_{2} X+s_{3} Y+s_{4} X^{2}$, then $\beta$ admits a $\Gamma$-representing measure if and only if the relations $X^{3}=Y, X^{2} Y=r_{1} X+r_{2} X^{2}+r_{3} X Y+r_{4} Y, X Y^{2}=$ $r_{1} Y+r_{2} X Y+r_{3} Y^{2}+r_{4} X^{2} Y$, and $Y^{3}=s_{1} Y+s_{2} X Y+s_{3} Y^{2}+s_{4} X^{2} Y$ unambiguously define a flat moment matrix extension $M(3)$.

iv) If rank $M(2)=5$ and there is a column relation $X Y=r_{1} 1+r_{2} X+r_{3} Y+$ $r_{4} X^{2}$, then there is no $\Gamma$-representing measure.

v) If rank $M(2)=5$ and there is a column relation $Y^{2}=s_{1} 1+s_{2} X+s_{3} Y+s_{4} X^{2}+$ $s_{5} X Y$, then there is a $\Gamma$-representing measure if and only if the relations $X^{3}=Y$, $X Y^{2}=s_{1} X+s_{2} X^{2}+s_{3} X Y+s_{4} X^{3}+s_{5} X^{2} Y$, and $Y^{3}=s_{1} Y+s_{2} X Y+s_{3} Y^{2}+s_{4} X^{2} Y$ unambiguously define a positive extension $M(3)$ and recursively determine a flat extension $M(4)$.

Proof. The proof of i) is an immediate application of Theorem 1.6. For the remaining cases, we recall that if $\beta$ has a $\Gamma$-representing measure, then there exists a finitely atomic $\Gamma$-representing measure $\nu$. For ii), note that if there exists a $\Gamma$ representing measure, then in $M(3)[\nu]$ we must have $X^{2}=d_{1} 1+d_{2} X+d_{3} X^{3}$, whence card supp $\nu \leq 3<\operatorname{rank} M(2) \leq \operatorname{rank} M(3)[\nu]$, a contradiction. To prove iii), note that if $\beta$ admits a $\Gamma$-representing measure, then the indicated column relations hold in $M(3)[\nu]$. Conversely, if these column relations unambiguously define a moment matrix extension $M(3)$, then $M(3)$ is a flat extension whose corresponding representing measure is necessarily supported in $\Gamma$. For part iv), if there were a $\Gamma$-representing measure, then in $M(4)[\nu]$ there would be a relation $X^{4}=r_{1} 1+r_{2} X+r_{3} X^{3}+r_{4} X^{2}$, whence card supp $\nu \leq 4<\operatorname{rank} M(4)[\nu]$, a contradiction. In case v), if there is a $\Gamma$-representing measure, then $M(3)[\nu]$ would be determined by the indicated column relations, and these relations would recursively determine $M(4)[\nu]$ as well. Further, since supp $\nu$ is contained in the intersection of the curves $y^{2}=s_{1}+s_{2} x+s_{3} y+s_{4} x^{2}$ and $y=x^{3}$, then $5=$ $\operatorname{rank} M(2) \leq \operatorname{rank} M(3)[\nu] \leq \operatorname{rank} M(4)[\nu] \leq$ card supp $\nu \leq 6$. Thus, either $\operatorname{rank} M(2)=\operatorname{rank} M(3)[\nu]$, and thus also $\operatorname{rank} M(3)[\nu]=\operatorname{rank} M(4)[\nu]$, or $\operatorname{rank} M(2)<\operatorname{rank} M(3)[\nu]=\operatorname{rank} M(4)[\nu]$; in either case, $M(4)[\nu]$ is a flat extension of $M(3)[\nu]$. Conversely, if $M(3)$ and $M(4)$ are well-defined by the indicated column relations and $M(4)$ is a flat extension of $M(3)$, then clearly $\beta$ has a $\Gamma$-representing measure.

In case $\mathrm{v})$, if $M(3)$ is a flat extension of $M(2)$, there is no need to consider $M(4)$. However, examples show that a 2 -step extension process may be needed if card $\mathcal{V}(M(2))=6$, which occurs when $\mathcal{V}(M(2))$ is the 6-point intersection of a degenerate hyperbola (2 intersecting lines) with $y=x^{3}$.

We next address the case where $M(2)$ is positive and $M(1)$ is singular.

Proposition 5.8. For $\beta \equiv \beta^{(4)}$, suppose $M(2)$ is positive and recursively generated and $M(1)$ is singular.

i) If rank $M(1)=1$, then $\beta$ has a $\Gamma$-representing measure if and only if $b=a^{3}$.

ii) If $\operatorname{rank} M(1)=\operatorname{rank} M(2)=2$, then $\beta$ has a $\Gamma$-representing measure if and only if the unique (2-atomic) representing measure associated with the flat extension $M(2)$ is supported in $\Gamma$.

iii) If neither i) nor ii) hold, then rank $M(1)=2$ and $\operatorname{rank} M(2)=3$. In this case, if $\mathcal{S} \equiv\left\{1, X, X^{2}\right\}$ is dependent, there is no $\Gamma$-representing measure. If 
$\mathcal{S}$ is independent, there is a column relation $Y=r 1+s X$. Then $\beta$ has a $\Gamma$ representing measure if and only if the relations $X^{3}=Y, X^{2} Y=r X^{2}+s Y$, $X Y^{2}=r X Y+s X^{2} Y$, and $Y^{3}=r Y^{2}+s X Y^{2}$ define a flat extension $M(3)$.

Proof. Since $\beta_{0,0}>0$, if $\operatorname{rank} M(1)=1$, we have $X=a 1$ and $Y=b 1$, and it follows from recursiveness that there is a unique representing measure, with support at $(a, b)$. Thus, there is a $\Gamma$-representing measure if and only if $b=a^{3}$. The proof of ii) is clear, since the measure corresponding to the flat extension $M(2)$ of $M(1)$ is the unique representing measure for $\beta$ (cf. Theorem 1.6). For iii), recursiveness implies rank $M(2)=3$. Suppose there is a column relation $X^{2}=r 1+s X$. If $\beta$ admits a $\Gamma$-representing measure, then there is a finitely atomic $\Gamma$-representing measure $\nu$, and in $M(3)[\nu]$ there are relations $Y=X^{3}$ and $X^{2}=r 1+s X$. These relations imply card supp $\nu \leq 2$, whence $3=\operatorname{rank} M(2) \leq \operatorname{rank} M(3)[\nu] \leq$ card supp $\nu \leq 2$, a contradiction. In the remaining case, since $M(1)$ is singular and $\{1, X\}$ is independent, there is a relation $Y=r 1+s X$. If $\beta$ admits a $\Gamma$ representing measure, then, as above, in $M(3)[\nu]$, there are relations $X^{3}=Y$, $X^{2} Y=r X^{2}+s Y, X Y^{2}=r X Y+s X^{2} Y$, and $Y^{3}=r Y^{2}+s X Y^{2}$, so $M(3)[\nu]$ is a flat extension of $M(2)$. Conversely, if these relations define a flat extension $M(3)$, then the representing measure corresponding to $M(3)$ via Theorem 1.6 is necessarily supported in $\Gamma$ (since $\left.Y=X^{3}\right)$.

Finally, we consider the case when $n=1$ and $M(1)$ is positive and singular.

Proposition 5.9. Let $\beta \equiv \beta^{(2)}$ and suppose $M(1)$ is positive and singular.

i) If $\operatorname{rank} M(1)=1$, then $\beta$ has a $\Gamma$-representing measure if and only if $b=a^{3}$.

ii) If $\operatorname{rank} M(1)=2$ and $\{1, X\}$ is dependent, there is no $\Gamma$-representing measure.

iii) If neither $i)$ nor ii) hold, then there is a relation $Y=r 1+s X$. Let $\sigma:=$ $\left\{\left(x, x^{3}\right): x^{3}=r+s x\right\}$. If card $\sigma=1, \beta$ has no $\Gamma$-representing measure. If card $\sigma=2$, there is a unique 2-atomic measure $\mu_{1}$, supp $\mu_{1}=\sigma$, such that $\beta$ has a $\Gamma$-representing measure if and only if $\mu_{1}$ is a $\Gamma$-representing measure. If card $\sigma=3$, there are three 2-atomic measures, $\mu_{i}(1 \leq i \leq 3)$, with supp $\mu_{i} \subset \sigma$, and at most one 3-atomic measure $\mu_{4}$, supp $\mu_{4}=\sigma$, such that $\beta$ has a $\Gamma$-representing measure if and only if some $\mu_{i}$ is a $\Gamma$-representing measure.

Proof. The proof of i) is similar to the proof of Proposition 5.8-i). For ii), suppose $X=r 1$. As in the proof of Proposition 5.8, if $\nu$ is a finitely atomic $\Gamma$ representing measure, then in $M(3)[\nu]$ we have $Y=X^{3}, X=r 1$, whence $2=\operatorname{rank} M(1) \leq$ rank $M(3)[\nu] \leq$ card supp $\nu \leq 1$, a contradiction. In case iii), proceeding as above, in $M(3)[\nu]$ there is a column relation $X^{3}=r 1+s X$, whence supp $\nu \subseteq \sigma$ and card supp $\nu \leq 3$. If card $\sigma=1$, then as in ii) above, card supp $\nu \leq 1$, a contradiction, so there is no $\Gamma$-representing measure. Suppose card $\sigma=3, \sigma=$ $\left\{\left(x_{i}, y_{i}\right)\right\}_{1 \leq i \leq 3}$. Let $V$ be as in (5.17). If $V$ is invertible, we may define a measure $\mu_{4} \equiv \sum_{i=1}^{3} \rho_{i} \delta_{\left(x_{i}, y_{i}\right)}$, where $\rho \equiv\left(\rho_{1}, \rho_{2}, \rho_{3}\right)$ is defined as in (5.18). Then $\beta$ has a 3 -atomic $\Gamma$-representing measure if and only if $\mu_{4}$ is such a measure. If $V$ is singular, we test for a 2-atomic $\Gamma$-representing measure. Select any two distinct points of $\sigma, x_{i_{1}}$ and $x_{i_{2}}$, form $V=\left(\begin{array}{cc}1 & 1 \\ x_{i_{1}} & x_{i_{2}}\end{array}\right)$ (which is invertible), and use $\left(\rho_{1}, \rho_{2}\right)^{t}=V^{-1}(1, a)^{t}$ to form the associated measure $\mu \equiv \sum_{k=1}^{2} \rho_{k} \delta_{\left(x_{i_{k}}, y_{i_{k}}\right)}$. Then 
$\beta$ has a 2 -atomic $\Gamma$-representing measure if and only if some measure $\mu$ of this form is a $\Gamma$-representing measure. The case when card $\sigma=2$ is treated similarly.

\section{Representing MEASURES AND MOMENT MATRix EXTENSiOns}

In CF8 we studied polynomials $p$ for which the existence of representing measures in the truncated $\mathcal{Z}_{p}$-moment problem can be expressed in terms of moment matrix extensions, without the requirement for an ultimate flat extension as in Theorem 1.8. In the present section we give new examples of this phenomenon. In the sequel, we consider the general case, $d \geq 1$, unless otherwise noted. Recall from [S1] that in the full moment problem, $p \in \mathcal{P} \equiv \mathbb{R}\left[x_{1}, \ldots, x_{d}\right]$ is type $A$ if the conditions $M(\infty)(\beta) \succeq 0$ and $p(X)=0$ imply that $\beta^{(\infty)}$ admits a $\mathcal{Z}_{p}$-representing measure (these conditions are always necessary for a $\mathcal{Z}_{p}$-representing measure). In S1, Stochel showed that $p$ is type A if and only if the polynomials that are nonnegative on $\mathcal{Z}_{p}$ are in the closure of sums of squares modulo the principal ideal $(p)$ (with respect to a suitable topology). This result depends in part on the classical Riesz-Haviland Theorem $[\mathrm{H}]: \beta \equiv \beta^{(\infty)}$ has a $K$-representing measure if and only if $\Lambda_{\beta}: \mathcal{P} \longrightarrow \mathbb{R}$ is $K$-positive, i.e., $p \in \mathcal{P}, p \mid K \geq 0 \Longrightarrow \Lambda_{\beta}(p) \geq 0$. In [CF8], we recently obtained the following analogue of the Riesz-Haviland Theorem for the truncated $K$-moment problem: $\beta \equiv \beta^{(2 n)}$ has a $K$-representing measure if and only if $\Lambda_{\beta}: \mathcal{P}_{2 n} \longrightarrow \mathbb{R}$ admits a $K$-positive linear extension $\widetilde{\Lambda}: \mathcal{P}_{2 n+2} \longrightarrow \mathbb{R}$. Motivated by this result, we consider the following property for $p \in \mathcal{P}_{n}$; here $n \geq 1$ and $m \geq n+1$ :

$$
\left(A_{n, m}\right) \quad f \in \mathcal{P}_{2 n+2}, f \mid \mathcal{Z}_{p}>0 \Longrightarrow f=\sum f_{i}^{2}+p q,\left(f_{i}, q \in \mathcal{P}_{m}\right) .
$$

Proposition 6.1. Suppose $p \in \mathcal{P}_{n}$ satisfies $\left(A_{n, m}\right)$ for some $m \geq n+1$. Then $\beta \equiv \beta^{(2 n)}$ has a $\mathcal{Z}_{p}$-representing measure if and only if $p(X)=0$ in $\mathcal{C}_{M(n)}$ and $M(n)$ admits a positive extension $M(m)$. Further, if $f \in \mathcal{P}_{2 n}$ and $f \mid \mathcal{Z}_{p}>0$, then $f=\sum f_{i}^{2}+p \sum g_{i}^{2}-p \sum h_{i}^{2}\left(\operatorname{deg} f_{i}^{2}, \operatorname{deg} p g_{i}^{2}, \operatorname{deg} p h_{i}^{2} \leq 2 m\right)$.

Proof. Since $p \in \mathcal{P}_{n}$, if $\mu$ is a representing measure for $\beta$ with supp $\mu \subseteq \mathcal{Z}_{p}$, then (1.5) implies that $p(X)=0$ in $\mathcal{C}_{M(n)}$. Further, BT implies that there is a finitely atomic $\mathcal{Z}_{p}$-representing measure $\nu$ and that $M(m)[\nu] \succeq 0$.

Conversely, suppose $p(X)=0$ in $\mathcal{C}_{M(n)}$ and there is a positive extension $M(m)$, where $m \geq n+1$. We may consider $\widetilde{\Lambda} \equiv \Lambda_{\beta^{(2 n+2)}}: \mathcal{P}_{2 n+2} \longrightarrow \mathbb{R}$ and $\Lambda \equiv \Lambda_{\beta^{(2 m)}}$ : $\mathcal{P}_{2 m} \longrightarrow \mathbb{R}$. We claim that $\widetilde{\Lambda}$ is $\mathcal{Z}_{p}$-positive. Suppose $f \in \mathcal{P}_{2 n+2}, f \mid \mathcal{Z}_{p}>0$. From $\left(A_{n, m}\right)$, there exist polynomials $f_{1}, \ldots, f_{s}, q \in \mathcal{P}_{m}$ such that $f=\sum f_{i}^{2}+p q$. Now, $\widetilde{\Lambda}(f)=\Lambda(f)=\Lambda\left(\sum f_{i}^{2}+p q\right)=\sum\left\langle M(m) \widehat{f}_{i}, \widehat{f}_{i}\right\rangle+\langle M(m) \widehat{p}, \widehat{q}\rangle$. From the Extension Principle, $p(X)=0$ in $M(m)$, so $M(m) \widehat{p}=p(X)=0$, and thus $\widetilde{\Lambda}(f) \geq 0$. If $\epsilon>0$ and $f \in \mathcal{P}_{2 n+2}$ with $f \mid \mathcal{Z}_{p} \geq 0$, then $(f+\epsilon) \mid \mathcal{Z}_{p}>0$, whence (from above) $\widetilde{\Lambda}(f+\epsilon) \geq 0$. It follows that $\widetilde{\Lambda}(f) \geq 0$, so $\widetilde{\Lambda}$ is $\mathcal{Z}_{p}$-positive, and thus the Truncated Riesz-Haviland Theorem ([CF8, Theorem 1.2]) implies that $\beta$ admits a $\mathcal{Z}_{p}$-representing measure.

For the representation of positive polynomials, we view $\mathcal{Z}_{p}$ as a semialgebraic set. Let $\mathcal{Q} \equiv\left\{q_{j}\right\}_{j=0}^{t} \subset \mathcal{P}_{2 n}\left(q_{0}=1\right)$, and let $K_{\mathcal{Q}}$ denote the semialgebraic set $K_{\mathcal{Q}}=\left\{x \in \mathbb{R}^{d}: q_{j}(x) \geq 0,0 \leq j \leq t\right\}$. Let $\Sigma_{\mathcal{Q}, n}=\left\{p \in \mathcal{P}_{2 n}: p=\right.$ $\left.\sum f_{0, i}^{2}+q_{1} \sum f_{1, i}^{2}+\cdots+q_{t} \sum f_{t, i}^{2}, q_{j} f_{j, i}^{2} \in \overline{\mathcal{P}}_{2 n}(0 \leq j \leq t)\right\}$. For $k \geq 0$, consider property $\left(S_{n, k}\right)$ of [CF8 for $K_{\mathcal{Q}}$ : if $M(n)$ admits a positive extension $M(n+k)$ such that each localizing matrix $M_{q_{j}}(n+k)(0 \leq j \leq t)$ is positive, then $\beta^{(2 n)}$ has a 
$K_{\mathcal{Q}}$-representing measure (these conditions are always necessary). CF8, Theorem 1.5] shows that if $K_{\mathcal{Q}}$ satisfies $\left(S_{n, k}\right)$ for some $k \geq 0$, then each polynomial in $\mathcal{P}_{2 n}$ that is strictly positive on $K_{\mathcal{Q}}$ belongs to $\Sigma_{\mathcal{Q}, n+k}$. In what follows, we set $q_{0}=1$, $q_{1}=p, q_{2}=-p, Q=\left\{q_{0}, q_{1}, q_{2}\right\}$, and view $\mathcal{Z}_{p}$ as $K_{\mathcal{Q}}$. Now let $f \in \mathcal{P}_{2 n}$ with $f \mid \mathcal{Z}_{p}>0$. To establish the desired weighted sum-of-squares decomposition for $f$, we show that $\mathcal{Z}_{p}$ satisfies property $\left(S_{n, m-n}\right)$. Suppose $M(n)$ admits a positive extension $M(m)$ satisfying $M_{p}(m) \succeq 0$ and $M_{-p}(m) \succeq 0$. Then $M_{p}(m)=0$, and since $m \geq n+1$, [CF4, Prop. 3.9-i] implies that $p(X)=0$ in $\mathcal{C}_{M(m)}$. Since $p$ satisfies $\left(A_{n, m}\right)$, the existence of a $\mathcal{Z}_{p}$-representing measure now follows from the first part of the proof. Thus, $\mathcal{Z}_{p}$ satisfies $\left(S_{n, m-n}\right)$, so the representation for $f$ follows from CF8, Theorem 1.5].

Note that in Proposition 6.1 the weighted sum-of-squares representation for $f$ is degree-bounded in the sense that the bound, $2 m$, on the degrees of the summands in the representation is independent of $f$.

In [S1, Stochel proved that type A polynomials include the planar polynomials $p_{1}(x, y)=y-q(x), p_{2}(x, y)=y q(x)-1(q \in \mathbb{R}[x])$, and $p_{3}(x, y)$ with $\operatorname{deg} p_{3} \leq 2$. We can prove these results via the truncated moment problem by using moment matrix extensions. This was previously done for $p_{3}$ in CF7] (cf. Theorem 1.2). To treat $p_{1}$ and $p_{2}$, we require the following result.

Corollary 6.2. Let $p \in \mathcal{P}$. If for each $n \geq \operatorname{deg} p$ there exists $m(n) \geq n+1$ such that $p$ satisfies $\left(A_{n, m(n)}\right)$, then $p$ is type $A$.

Proof. Suppose the hypothesis holds and suppose $M(\infty)(\beta) \succeq 0$ and $p(X)=0$ in $\mathcal{C}_{M(\infty)}$. For each $n \geq \operatorname{deg} p, p(X)=0$ in $M(n)$ and $M(m(n))$ is a positive extension of $M(n)$, so Proposition 6.1 implies that $\beta^{(2 n)}$ has a $\mathcal{Z}_{p^{-} \text {-representing measure. It }}$

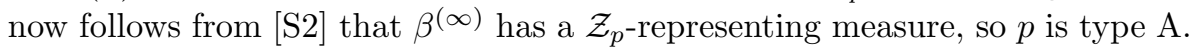

We now consider $d=2$ and $p_{1}(x, y)=y-q(x)$ for some $q \in \mathbb{R}[x]$, deg $q \geq 1$.

Proposition 6.3. Let $p \equiv p_{1}$. For $n \geq \operatorname{deg} p$, $p$ satisfies $\left(A_{n, m}\right)$ with $m=$ $(2 n+2) \operatorname{deg} q-\operatorname{deg} p$.

Proof. The proof follows the argument in [S1, Prop. 5.2], except that we also keep track of the degrees of the polynomials that appear. Suppose $f(x, y) \in \mathcal{P}_{2 n+2}$ and $f \mid \mathcal{Z}_{p}>0$. Then $s(x):=f(x, q(x))>0(x \in \mathbb{R})$ and deg $s \leq(2 n+2) \operatorname{deg} q$. [PoSz, p. 77] implies that there are polynomials $g(x)$ and $h(x)$ such that $s(x)=$ $g(x)^{2}+h(x)^{2}(x \in \mathbb{R})$. Since $p$ is irreducible in $\mathbb{R}[x, y]$ and $\mathcal{Z}_{p}$ is infinite, then $I\left(\mathcal{Z}_{p}\right) \equiv\left\{u(x, y) \in \mathcal{P}: u \mid \mathcal{Z}_{p} \equiv 0\right\}=(p)$ (cf. [S1, Prop. 3.4]), so there is a polynomial $r(x, y)$ such that $f-g^{2}-h^{2}=p r$. Further, if $\rho:=(2 n+2) \operatorname{deg} q-\operatorname{deg} p$, then $\operatorname{deg} g, \operatorname{deg} h \leq(n+1) \operatorname{deg} q \leq \rho$ and $\operatorname{deg} r \leq \rho$. Since $\rho \geq n+1$, then $p$ satisfies $\left(A_{n, m}\right)$ with $m=\rho$.

Note that for $p(x, y)=y-x^{3}$, Propositions 6.1 and 6.3 imply that $\beta^{(2 n)}$ has a $\Gamma$-representing measure if $Y=X^{3}$ in $\mathcal{C}_{M(n)}$ and $M(n)$ admits a positive extension $M(6 n+3)$. By contrast, Corollary 5.3 implies that there is a $\Gamma$-representing measure if $M(n)$ merely has a positive extension $M(n+3)$ (so that $M(n+2)$ is positive and recursively generated; cf. [CF2, Theorem 3.14].

We now turn to $p_{2}(x, y)=y q(x)-1$ with $q \in \mathbb{R}[x], \operatorname{deg} q \geq 1$.

Proposition 6.4. Let $p=p_{2}$. For $n \geq \operatorname{deg} p$, $p$ satisfies $\left(A_{n, m}\right)$ with $m=$ $(2 n+2)(2+\operatorname{deg} q)-(1+\operatorname{deg} q)$. 
Proof. We again adapt the proof of [S1, Prop. 5.2]. Suppose $f(x, y) \in \mathcal{P}_{2 n+2}$ and $f \mid \mathcal{Z}_{p}>0$. Writing $f(x, y)=\sum_{i, j \geq 0, i+j \leq 2 n+2} a_{i j} x^{i} y^{j}$, it follows that $s(x):=$ $\sum_{i, j \geq 0, i+j<2 n+2} a_{i j} x^{i} q(x)^{2 n+2-j}$ satisfies $s(x) \geq 0(x \in \mathbb{R})$ and $\operatorname{deg} s \leq(2 n+$ 2)deg $q$. Thus, from $\mathrm{PoSz}$, there are polynomials $g(x), h(x)$, with $\operatorname{deg} g$, deg $h \leq$ $(n+1) \operatorname{deg} q$, such that $s(x)=g(x)^{2}+h(x)^{2}$. Let $G(x, y)=y^{n+1} g(x), H(x)=$ $y^{n+1} h(x)$, and $F(x, y)=f(x, y)-\left(G(x, y)^{2}+H(x, y)^{2}\right)$. We have $F \mid \mathcal{Z}_{p} \equiv 0$, and since $p$ is irreducible in $\mathbb{R}[x, y]$ and $\mathcal{Z}_{p}$ is infinite, it follows from [S1, Prop. 3.4] that $F=p r$ for some polynomial $r \equiv r(x, y)$. Thus, $f=G^{2}+H^{2}+p r$. Since $\operatorname{deg} f \leq$ $2 n+2$ and $\operatorname{deg}\left(G^{2}+H^{2}\right) \geq 2 n+2$, then $\operatorname{deg} p r \leq \operatorname{deg}\left(G^{2}+H^{2}\right) \leq(2 n+2)(1+\operatorname{deg} q)$, whence $\operatorname{deg} r \leq(2 n+2)(1+\operatorname{deg} q)-\operatorname{deg} p=(2 n+2)(1+\operatorname{deg} q)-(1+\operatorname{deg} q)=$ $(2 n+1)(1+\operatorname{deg} q) \equiv \tau$. Now, deg $G^{2} \leq 2 n+2+\operatorname{deg} g^{2} \leq 2 n+2+\operatorname{deg} s \leq$ $2 n+2+(2 n+2)(\operatorname{deg} q)$, so $\operatorname{deg} G \leq(n+1)(1+\operatorname{deg} q)<\tau$, and similarly for $H$. Thus, $p$ satisfies $\left(A_{n, m}\right)$ with $m=\tau$.

Remark 6.5. The preceding results imply that for $i=1,2, p_{i}$ is type A and each polynomial that is strictly positive in $\mathcal{Z}_{p_{i}}$ admits a degree-bounded weighted sumof-squares decomposition (as in Proposition 6.1).

\section{REFERENCES}

[Akh] N.I. Akhiezer, The Classical Moment Problem, Hafner Publ. Co., New York, 1965. MR0184042 (32:1518)

[AK] N.I. Ahiezer and M.G. Krein, Some Questions in the Theory of Moments, Trans. Math. Monographs, vol. 2, Amer. Math. Soc., Providence, RI, 1962. MR0167806 (29:5073)

[Atz] A. Atzmon, A moment problem for positive measures on the unit disc, Pacific J. Math. 59(1975), 317-325. MR0402057(53:5880)

[BM] C. Berg and P.H. Maserick, Polynomially positive definite sequences, Math. Ann 259(1982), 487-495. MR660043 (84k:44012)

[BT] C. Bayer and J. Teichmann, The proof of Tchakaloff's Theorem, Proc. Amer. Math. Soc. 134(2006), 3035-3040. MR2231629 (2007d:44004)

[BiS] T.M. Bisgaard and J. Stochel, Algebraic sets of types A, B and C coincide, preprint, 2007.

[Cas] G. Cassier, Problème des moments sur un compact de $\mathbb{R}^{n}$ et décompositions de polynômes a plusieurs variables, J. Funct. Anal. 58(1984), 254-266. MR.759099 (85m:44004)

[CF1] R. Curto and L. Fialkow, Recursiveness, positivity, and truncated moment problems, Houston J. Math 17(1991), 603-635. MR.1147276 (93a:47016)

[CF2] R. Curto and L. Fialkow, Solution of the truncated complex moment problem for flat data, Memoirs Amer. Math. Soc. 568(1996), x+52 pp. MR.1303090 (96g:47009)

[CF3] R. Curto and L. Fialkow, Flat extensions of positive moment matrices: Recursively generated relations, Memoirs Amer. Math. Soc. 136 (1998), no. 648, x + 56pp.

[CF4] R. Curto and L. Fialkow, The truncated complex $K$-moment problem, Trans. Amer. Math. Soc. 352(2000), 2825-2855. MR1661305 (2000j:47027)

[CF5] R. Curto and L. Fialkow, A duality proof of Tchakaloff's theorem, J. Math. Anal. Appl. 269(2002), 519-532. MR 1907129 (2003e:41047)

[CF6] R. Curto and L. Fialkow, Truncated $K$-moment problems in several variables, J. Operator Theory 54(2005), 189-226. MR2168867 (2006e:47032)

[CF7] R. Curto and L. Fialkow, Solution of the truncated hyperbolic moment problem, Integral Equations and Operator Theory 52(2005), 181-218. MR2216081 (2007a:47017)

[CF8] R. Curto and L. Fialkow, An analogue of the Riesz-Haviland theorem for the truncated moment problem, J. Functional Analysis 225(2008), 2709-2731. MR2464189 (2009i:47039)

[CFM] R. Curto, L. Fialkow, and H.M. Möller, The extremal truncated moment problem, Integral Equations and Operator Theory 60(2008), 177-200. MR2375563(2009b:47021a)

[D] R.G. Douglas, On majorization, factorization and range inclusion of operators on Hilbert spaces, Proc. Amer. Math. Soc. 17(1966), 413-415. MR0203464 (34:3315)

[F1] L. Fialkow, Positivity, extensions and the truncated complex moment problem, Contemporary Math. 185(1995), 133-150. MR1332058 (96g:44013) 
[F2] L. Fialkow, Minimal representing measures arising from rank-increasing moment matrix extensions, J. Operator Theory 42(1999), 425-436. MR.1717016 (2000f:47025)

[F3] L. Fialkow, Truncated complex moment problems with a $\bar{z} z$ relation, Integral Equations and Operator Theory 45(2003), 405-435. MR.1971746 (2004b:47015)

[F4] L. Fialkow, Truncated multivariable moment problems with finite variety, Journal of Operator Theory 60(2008), 343-377. MR2464216 (2009i:47040)

[Fug] B. Fuglede, The multidimensional moment problem, Exposition. Math. 1(1983), 47-65. MR0693807 (85g:44010)

[H] E.K. Haviland, On the momentum problem for distributions in more than one dimension II, Amer. J. Math. 58(1936), 164-168. MR.1507139

[KN] M.G. Krein and A. Nudel'man, The Markov Moment Problem and Extremal Problems, Transl. Math. Monographs, vol. 50, Amer. Math. Soc., Providence, 1977. MR0458081 (56:16284)

[L] M. Laurent, Revisiting two theorems of Curto and Fialkow on moment matrices, Proc. Amer. Math. Soc. 133(2005), 2965-2976. MR2159775 (2006d:47027)

[M] I.P. Mysovskikh, On Chakalov's Theorem, U.S.S.R. Comput. Math. and Math. Phys. 15(1975), 221-227; translation in Zh. Vychisl. Mat. i Mat. Fiz. 15(1975), 1589-1593.

[PoSz] G. Polya and G. Szego, Problems and Theorems in Analysis, Vol. II, Springer-Verlag, New York-Heidelberg-Berlin, 1976. MR0396134 (53:2)

[PS] V. Powers and C. Scheiderer, The moment problem for non-compact semialgebraic sets, Adv. Geom. 1(2001), 71-88. MR.1823953 (2002c:14086)

[P] M. Putinar, On Tchakaloff's Theorem, Proc. Amer. Math. Soc. 125(1997), 2409-2414. MR1389533 (97j:65045)

[PV] M. Putinar, F.-H. Vasilescu, Solving moment problems by dimensional extension, Ann. of Math. (2) 149(1999), no. 3, 1087-1107. MR.1709313(2001c:47023b)

[R] M. Riesz, Sur le problème des moments, Troisième Note, Arkiv für Matematik, Astronomi och Fysik 17(1923), 1-52.

[Sch1] K. Schmüdgen, The $K$-moment problem for semi-algebraic sets, Math. Ann. 289(1991), 203-206. MR:1092173 (92b:44011)

[Sch2] K. Schmüdgen, On the moment problem of closed semi-algebraic sets, J. Reine Angew. Math. 588(2003), 225-234. MR.1979186 (2004e:47019)

[ST] J. Shohat and J. Tamarkin, The Problem of Moments, Math. Surveys I, Amer. Math. Soc., Providence, 1943. MR.0008438 (5:5c)

[Smu] J.L. Smul'jan, An operator Hellinger integral (Russian), Mat.Sb. 91(1959), 381-430. MR0121662(22:12396)

[S1] J. Stochel, Moment functions on real algebraic sets, Arkiv for matematik 30(1992), 133148. MR1171099 (93d:47032)

[S2] J. Stochel, Solving the truncated moment problem solves the moment problem, Glasgow J. Math. 43(2001), 335-341. MR1878578 (2002j:47026)

[SS1] J. Stochel and F.H. Szafraniec, Algebraic operators and moments on algebraic sets, Portugal. Math. 51(1994), 25-45. MR.1281954 (95d:47013)

[SS2] J. Stochel and F.H. Szafraniec, The complex moment problem and subnormality: A polar decomposition approach, J. Funct. Anal. 159(1998), 432-491. MR.1658092 (2001c:47023a)

[T] V. Tchakaloff, Formules de cubatures mécaniques à coefficients non négatifs, Bull. Sc. Math. 81(1957), 123-134. MR0094632 (20:1145)

Department of Computer Science, State University of New York, New Paltz, New YORK 12561

E-mail address: fialkowl@newpaltz.edu 